TRANSACTIONS OF THE

AMERICAN MATHEMATICAL SOCIETY

Volume 359, Number 9, September 2007, Pages 4511-4535

S 0002-9947(07)04304-8

Article electronically published on April 17, 2007

\title{
THE ODD PRIMARY $H$-STRUCTURE OF LOW RANK LIE GROUPS AND ITS APPLICATION TO EXPONENTS
}

\author{
STEPHEN D. THERIAULT
}

\begin{abstract}
A compact, connected, simple Lie group $G$ localized at an odd prime $p$ is shown to be homotopy equivalent to a product of homotopy associative, homotopy commutative spaces, provided the rank of $G$ is low. This holds for $S U(n)$, for example, if $n \leq(p-1)(p-3)$. The homotopy equivalence is usually just as spaces, not multiplicative spaces. Nevertheless, the strong multiplicative features of the factors can be used to prove useful properties, which after looping can be transferred multiplicatively to $\Omega G$. This is applied to prove useful information about the torsion in the homotopy groups of $G$, including an upper bound on its exponent.
\end{abstract}

\section{InTRODUCTION AND STATEMENT OF RESUltS}

The compact, connected, simple Lie groups are $\operatorname{Spin}(n)$ for $n \geq 3, S U(n)$ for $n \geq 2, S p(n)$ for $n \geq 1$, and the exceptional groups $G_{2}, F_{4}, E_{6}, E_{7}$, and $E_{8}$. The group $\operatorname{Spin}(n)$ is the universal cover of $S O(n)$. The multiplicative properties of these groups from a homotopy theoretic point of view have been well investigated. Each has a classifying space and so is a loop space. In particular, all are homotopy associative. James and Thomas [JT] showed that none of them is homotopy commutative by showing that any Lie group whose loop multiplication is homotopy commutative must be homotopy equivalent to a torus. Bott $[\mathrm{B}$ made this explicit in the cases of $S U(n)$ and $S p(n)$ by exhibiting nontrivial Samelson products. Let $i \leq n$ and $x_{i} \in \pi_{2 i-1}(S U(n))=\mathbb{Z}$ represent a generator. Then if $i+j-1=n$, the Samelson product $\left\langle x_{i}, x_{j}\right\rangle$ has order $\frac{(i+j-1) !}{(i-1) !(j-1) !}$. A similar formula holds for $\operatorname{Sp}(n)$.

A natural question to ask is whether noncommutativity changes after localizing at a prime $p$. Bott's result shows that if $2 n>p$, then $i$ and $j$ can be chosen so that $\frac{(i+j-1) !}{(i-1) !(j-1) !}$ is divisible by $p$. Thus $\left\langle x_{i}, x_{j}\right\rangle$ is not null homotopic and so $S U(n)$ for $2 n>p$ is not homotopy commutative when the loop multiplication has been localized. The analogous formula for $S p(n)$ gives a similar result. McGibbon $[\mathrm{Mc}$ classified all the compact, connected, simple Lie groups $G$ whose loop multiplications are homotopy commutative when localized at $p$. They are: $\operatorname{Spin}(2 n+1)$ if $4 n<p, \operatorname{Spin}(2 n)$ if $4(n-1)<p, S U(n)$ if $2 n<p, S p(n)$ if $4 n<p, S p(2)$ at the prime 3 , and $G_{2}$ at the prime 5 . On the other hand, the situation changes if nonloop multiplications are considered. McGibbon $\mathrm{Mc}$ and Iriye and Kono $\mathrm{IK}$ showed that at odd primes there is always some multiplication on $G$ which is

Received by the editors October 18, 2005.

2000 Mathematics Subject Classification. Primary 55P45, 55Q52, 57T20.

Key words and phrases. Lie group, exponent, Whitehead product, $H$-space. 
homotopy commutative. However, this exotic multiplication is not known to be homotopy associative, and except for special cases, probably is not.

In this paper we seek to combine the properties of homotopy associativity and homotopy commutativity. We show that in low rank, a compact, connected, simple Lie group $G$ is homotopy equivalent to an $H$-space which is both homotopy associative and homotopy commutative. At first glance this is a strange statement, because the homotopy equivalence is not as $H$-spaces, it is only as spaces. So we are not talking about a multiplication directly on $G$ but rather one on a homotopy equivalent space. Nevertheless, this "other" space arises naturally, and the associative and commutative properties of its multiplication allow us to prove interesting properties about the homotopy and multiplicative exponents of $G$ - discussed in this paper - and the subgroup of multiplicative self-maps in $[G, G]$ - to be discussed in subsequent work.

The "other" space is going to be a certain universal space. We begin by defining what is meant by universal. Suppose there is a map $i: A \longrightarrow B$ where $A$ is a space and $B$ is a homotopy associative, homotopy commutative $H$-space. We say that $B$ is universal for $A$ if any map $f: A \longrightarrow Z$ into a homotopy associative, homotopy commutative $H$-space $Z$ extends to a unique $H$-map $\bar{f}: B \longrightarrow Z$ with the property that $f \simeq \bar{f} \circ i$. (See Section 3 for a general discussion and examples.) There is no known functorial construction of universal spaces; the known examples are constructed on a case by case basis. Typically, starting with a space $A$ one looks for an $H$-space $B$ with $H_{*}(B) \cong S\left(\widetilde{H}_{*}(A)\right)$, and hopes to show that $B$ is universal for $A$. Here, homology has been taken with mod- $p$ coefficients and $S()$ is the free symmetric algebra functor. For example, we have $H_{*}(S U(n)) \cong S\left(\widetilde{H}_{*}\left(\Sigma \mathbb{C} P^{n-1}\right)\right)$, and the canonical map $\Sigma \mathbb{C} P^{n-1} \longrightarrow S U(n)$ induces the inclusion of generating set in homology. But $S U(n)$ is not homotopy commutative if $2 n>p$, so in those cases it cannot be universal for $\Sigma \mathbb{C} P^{n-1}$. What we aim to do is construct the universal space for $\Sigma \mathbb{C} P^{n-1}$, provided $n \leq(p-1)(p-3)$, and show it is homotopy equivalent to $S U(n)$. This is the thrust of Theorem 1.1 when $G=S U(n)$.

To state the general case, recall that $G$ is rationally homotopy equivalent to a product of spheres, $G \simeq_{\mathbb{Q}} \prod_{j=1}^{l} S^{2 n_{j}-1}$, where $n_{1}<n_{2}<\ldots<n_{l}$. We say that the type of $G$ is $\left\{2 n_{1}-1, \ldots, 2 n_{l}-1\right\}$. Now localize at an odd prime $p$ and take homology with mod- $p$ coefficients. In a way that will be made more explicit in Section 2, there is a space $A(G)$ such that $H_{*}(G) \cong S\left(\widetilde{H}_{*}(A(G))\right)$. For each $G$, there is a homotopy decomposition $A(G) \simeq \bigvee_{i=1}^{p-1} A_{i}(G)$ where $\widetilde{H}_{*}\left(A_{i}(G)\right)$ consists of those elements in $\widetilde{H}_{*}(A(G))$ in degrees $2 i+2 k(p-1)+1$ for $0 \leq k<\infty$. Note that it may be possible for a given $G$ that $\widetilde{H}_{*}\left(A_{i}(G)\right)=0$ and so $A_{i}(G) \simeq *$. We show:

Theorem 1.1. Suppose $G$ is one of the following: $S U(n)$ if $n \leq(p-1)(p-3)$, $S p(n)$ if $2 n \leq(p-1)(p-3), \operatorname{Spin}(n)$ if $2 n+1 \leq(p-1)(p-3) ; G_{2}, F_{4}$, or $E_{6}$ if $p \geq 5 ; E_{7}$ or $E_{8}$ if $p \geq 7$. Then there is a homotopy decomposition

$$
G \simeq \prod_{i=1}^{p-1} B_{i}(G)
$$

where each $B_{i}(G)$ is homotopy associative, homotopy commutative, and universal for $A_{i}(G)$, and the product $\prod_{i=1}^{p-1} B_{i}(G)$ is universal for $A(G)$. 
A few brief remarks are in order. First, the restrictions on the primes imply that the $p=3$ case never arises, so we may as well assume from now on that $p \geq 5$. Second, if $G=S U(n)$, then $A(G)=\Sigma \mathbb{C} P^{n-1}$, and if $G=S p(n)$, then $A(G)=\Sigma \mathbb{H} P^{n-1}$. Third, if the decomposition of $A(G)$ has $A_{i}(G) \simeq *$ for some $i$, then $B_{i}(G) \simeq *$. Fourth, Mimura, Nishida, and Toda MNT2 gave explicit decompositions for every $G$ as a product $G \simeq \prod_{i=1}^{p-1} \bar{B}_{i}(G)$ where $H_{*}\left(\bar{B}_{i}(G)\right) \cong$ $S\left(\widetilde{H}_{*}\left(A_{i}(G)\right)\right)$ and each factor $\bar{B}_{i}(G)$ is indecomposable. In the decompositions of Theorem 1.1. the factors $B_{i}(G)$ are obtained differently, as applications of Cohen and Niesendorfer's [CN] construction of finite torsion free $H$-spaces. So $\bar{B}_{i}(G)$ and $B_{i}(G)$ may not be the same space, but we will show in Lemma 4.5 that they are homotopy equivalent.

In the cases where McGibbon proved the loop multiplication on $G$ is homotopy commutative, Theorem 1.1 improves. The universal property of $\prod_{i=1}^{p-1} B_{i}(G)$ for $A(G)$ implies that the usual inclusion $A(G) \longrightarrow G$ extends to an $H$-map $e: \prod_{i=1}^{p-1} B_{i}(G) \longrightarrow G$. Since $H_{*}\left(\prod_{i=1}^{p-1} B_{i}(G)\right) \cong S\left(\widetilde{H}_{*}(A(G))\right) \cong H_{*}(G)$ and $e_{*}$ is an algebra map which is an isomorphism on the generators, $e_{*}$ is an isomorphism and therefore $e$ is a homotopy equivalence. Thus:

Theorem 1.2. Let $p \geq 5$. Let $G$ be one of $\operatorname{Spin}(2 n+1)$ if $4 n<p$, $\operatorname{Spin}(2 n)$ if $4(n-1)<p, S U(n)$ if $2 n<p, S p(n)$ if $4 n<p$, or $G_{2}$ at the prime 5 . Then the decomposition in Theorem 1.1 is a homotopy equivalence of $H$-spaces. Consequently, in those cases, $G$ is universal for $A(G)$.

An interesting and practical example is $S U(3)$. It is well known [MT that $S U(3)$ is homotopy equivalent to $S^{3} \times S^{5}$ at primes $p \geq 3$. Theorem 1.2 shows that this homotopy equivalence is multiplicative if $p \geq 7$.

The decomposition in Theorem 1.1 and the universal property of the factors $B_{i}$ can be applied to prove exponent information about the group $G$. To set the stage, first return to Mimura, Nishida, and Toda's decomposition

$$
G \simeq \prod_{i=1}^{p-1} \bar{B}_{i}(G)
$$

. We say that $\bar{B}_{i}(G)$ has type $\left\{2 m_{1}-1, \ldots, 2 m_{k}-1\right\}$ if $\bar{B}_{i}(G)$ is rationally equivalent to $\prod_{j=1}^{k} S^{2 m_{j}-1}$, where $m_{1}<m_{2}<\ldots<m_{k}$. The same definition applies to $B_{i}(G)$. Mimura, Nishida, and Toda show that each $\bar{B}_{i}(G)$ is spherically resolved. In particular, there is a map to the highest dimensional resolving sphere $\bar{q}: \bar{B}_{i}(G) \longrightarrow$ $S^{2 m_{k}-1}$. As well, they show there is an integral summand in $\pi_{2 m_{k}-1}\left(\bar{B}_{i}(G)\right)$. Let $\bar{c}: S^{2 m_{k}-1} \longrightarrow \bar{B}_{i}(G)$ be a representative of a generator. Then $\bar{q} \circ \bar{c}$ has degree $p^{r_{i}(G)}$ for some nonnegative integer $r_{i}(G)$. In the case $G=S U(n)$, the map $\bar{c}$ is the standard characteristic map $S^{2 n-1} \longrightarrow S U(n)$ composed with the projection to the factor $\bar{B}_{i}(S U(n))$ whose type includes $2 n-1$. In this case, $r_{i}(G)=\nu_{p}((n-1) !)$, where $\nu_{p}(t)$ is the number of powers of $p$ which divide the positive integer $t$. Now switch to the decomposition $G \simeq \prod_{i=1}^{p-1} B_{i}(G)$ in Theorem 1.1. In Corollary 5.5 we show that $B_{i}(G)$ is spherically resolved, so there is a map to the highest dimensional resolving sphere $q: B_{i}(G) \longrightarrow S^{2 m_{k}-1}$. The homotopy equivalence $\bar{B}_{i}(G) \simeq B_{i}(G)$ composed with $\bar{c}$ gives a map $c: S^{2 m_{k}-1} \longrightarrow B_{i}(G)$. The composite $q \circ c$ has degree $p^{r_{i}(G)}$. Using the universal property of $B_{i}(G)$ we show that multiplication by $p^{r_{i}(G)}$ on $B_{i}(G)$ factors multiplicatively through the product of the resolving spheres. 
Theorem 1.3. Let $G$ be one of the groups listed in Theorem 1.1. Then there is a homotopy commutative diagram of $H$-maps

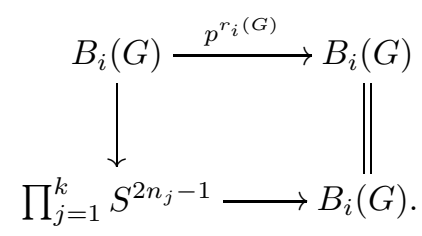

Moreover, with $H_{*}\left(B_{i}(G)\right) \cong S\left(\widetilde{H}_{*}\left(A_{i}(G)\right)\right)$, the exponent $r_{i}(G)$ is equal to the number of generators in $\widetilde{H}_{*}\left(A_{i}(G)\right)$ on which the dual Steenrod operation $\mathcal{P}_{*}^{1}$ acts nontrivially.

Now consider $G$ itself. As $G \simeq \prod_{i=1}^{p-1} B_{i}(G)$ and each $B_{i}(G)$ is spherically resolved, so is $G$. As the homotopy equivalence is not necessarily multiplicative, we cannot immediately combine the factorizations in Theorem 1.3 for each $B_{i}(G)$. But after looping, the multiplication on $\Omega G$ is an $H$-map, so the composite

$$
\prod_{i=1}^{p-1} \Omega B_{i}(G) \longrightarrow \prod_{i=1}^{p-1} \Omega G \stackrel{\mu}{\longrightarrow} \Omega G
$$

is a multiplicative homotopy equivalence. Suppose $G$ has type $\left\{2 n_{1}-1, \ldots, 2 n_{l}-1\right\}$. Let

$$
r(G)=\max \left\{r_{1}(G), \ldots, r_{p-1}(G)\right\} .
$$

Then multiplication by $p^{r(G)}$ on $\Omega G$ factors through the product of its looped resolving spheres. Specifically:

Theorem 1.4. Let $G$ be one of the groups listed in Theorem 1.1. Then there is a homotopy commutative diagram

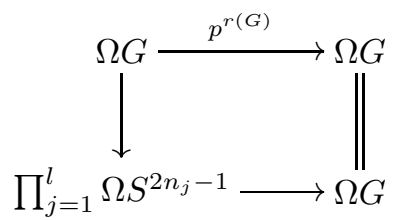

in which all maps are loop maps.

For example, when $G=S U(n)$, then the description of $r_{i}(S U(n))$ implies that $r(G)=\nu_{p}((n-1) !)$.

The factorization in Theorem 1.4 results in exponent information about $G$. Two types of exponents are considered. The homotopy exponent of a space $X$ is the least power of $p$ which annihilates the $p$-torsion in $\pi_{*}(X)$. We write this as $\exp (X)=p^{r}$. A stronger notion is that of a multiplicative exponent. If $Y$ is an $H$-space, then it has a $p^{t h}$-power map $p: Y \longrightarrow Y$. The multiplicative exponent of $Y$ is the least power of $p$ such that $Y \stackrel{p^{r}}{\longrightarrow} Y$ is null homotopic while $Y \stackrel{p^{r-1}}{\longrightarrow} Y$ is essential. We say that $Y$ has no multiplicative exponent if the $p^{r}$-power map on $Y$ is essential for all $r \in \mathbb{N}$. The notion of a multiplicative exponent is stronger than that of a homotopy exponent, for if the multiplicative exponent of $Y$ is $p^{r}$, then $\exp (Y) \leq$ $p^{r}$. A fundamental homotopy exponent result is due to [CMN, who showed that $\exp \left(S^{2 n-1}\right)=p^{n-1}$. Observe that the integral class in $\pi_{2 n-1}\left(S^{2 n-1}\right)$ guarantees that $S^{2 n-1}$ has no multiplicative exponent. To get this out of the way, consider the 
$(2 n-1)$-connected cover of $S^{2 n-1}$, denoted by $S^{2 n-1}\langle 2 n-1\rangle$. In NS] it was shown that $\Omega^{j} S^{2 n-1}\langle 2 n-1\rangle$ has no multiplicative exponent for $0 \leq j \leq 2 n-4$. When $p \geq 5$, it was shown in S2] that $\Omega^{2 n-3} S^{2 n-1}\langle 2 n-1\rangle$ has multiplicative exponent $p^{n-1}$. The factorization in Theorem 1.4 then implies the following:

Theorem 1.5. Suppose $G$ is one of the groups listed in Theorem 1.1, with type $\left\{2 n_{1}-1, \ldots, 2 n_{l}-1\right\}$. Then the following hold:

(a) $\Omega^{j} G\left\langle 2 n_{l}-1\right\rangle$ has no multiplicative exponent for $0 \leq j \leq 2 n_{l}-4$,

(b) $\Omega^{2 n_{l}-3} G\left\langle 2 n_{l}-1\right\rangle$ has multiplicative exponent $\leq p^{n_{l}-1+r(G)}$,

(c) $\exp (G) \leq p^{n_{l}-1+r(G)}$.

For example, when $G=S U(n)$, then $r(S U(n))=\nu_{p}((n-1) !)$ and so $\exp (S U(n))$ $\leq p^{n-1+\nu_{p}((n-1) !)}$.

Using different methods, the homotopy exponent bounds in Theorem 1.5 (c) were also obtained in $[\mathrm{DT}]$. The methods there, in fact, give exponent bounds for a wider range of Lie groups than those listed in Theorem 1.1. For example, the formula in Theorem 1.5 (c) is valid for $G=S U(n)$ if $n<p^{2}+p$ instead of just for $n \leq(p-1)(p-3)$. This alternative method has the advantage of being much simpler to compute, but the disadvantage of being more crude. It simply produces an upper bound on the homotopy exponent. It is not designed to track with precision how the torsion in the homotopy groups of $B_{i}(G)$ or $G$ are affected upon successive multiplications by $p$, in contrast to the factorizations in Theorems 1.3 and 1.4. The additional information these factorizations provide should be useful in other explicit calculations of the homotopy groups of $G$.

The author would like to thank Mamoru Mimura, who suggested that a paper originally about $S U(n)$ should really be about any compact, connected, simple Lie group $G$.

\section{Decompositions of Lie Groups}

In this section we review the known odd primary homotopy decompositions of compact, connected, simple Lie groups. We first consider the matrix groups and then the exceptional groups.

Harris [H] showed there are $p$-local homotopy equivalences

$$
\begin{gathered}
S U(2 n) \simeq S p(n) \times(S U(2 n) / S p(n)), \\
\operatorname{Spin}(2 n+1) \simeq S p(n), \\
\operatorname{Spin}(2 n) \simeq \operatorname{Spin}(2 n-1) \times S^{2 n-1} .
\end{gathered}
$$

Thus if $S U(n)$ could be written as a product of indecomposable factors for any $n$, Harris' equivalences would show that any matrix group could be written as a product of indecomposable factors. Such a decomposition of $S U(n)$ was obtained by Mimura, Nishida, and Toda MNT2. They used idempotents derived from unstable Adams operations to show that there is a $p$-local homotopy equivalence

$$
S U(n) \simeq \prod_{i=1}^{p-1} \bar{B}_{i}^{k}
$$

where each $\bar{B}_{i}^{k}$ is indecomposable and is described homologically as follows. Let $q=2(p-1)$. Take homology with mod- $p$ coefficients. Recall that $H_{*}(S U(n)) \cong$ 
$\Lambda\left(x_{3}, \ldots, x_{2 n-1}\right)$, where the degree of $x_{t}$ is $t$. Let $k=\left\lfloor\frac{n-i-1}{p-1}\right\rfloor+1$, where \lfloor\rfloor is the greatest integer less than or equal to. Then

$$
H_{*}\left(\bar{B}_{i}^{k}\right) \cong \Lambda\left(x_{2 i+1}, x_{2 i+q+1}, \ldots, x_{2 i+(k-1) q+1}\right) .
$$

The action of the dual Steenrod algebra is given by $\mathcal{P}_{*}^{j}\left(x_{2 r+1}\right)=\left(\begin{array}{l}r \\ j\end{array}\right) x_{2 r+j q+1}$.

Mimura, Nishida, and Toda then showed that this decomposition of $S U(n)$ together with Harris' decompositions give homotopy equivalences,

$$
\begin{gathered}
S p(n) \simeq \operatorname{Spin}(2 n+1) \simeq \prod_{i=1}^{(p-1) / 2} \bar{B}_{2 i-1}^{k^{\prime}}, \\
\operatorname{Spin}(2 n) \simeq S^{2 n-1} \times \prod_{i=1}^{(p-1) / 2} \bar{B}_{2 i-1}^{k^{\prime \prime}}
\end{gathered}
$$

where $k^{\prime}=\left\lfloor\frac{2(n-i)}{p-1}\right\rfloor+1$ and $k^{\prime \prime}=\left\lfloor\frac{2(n-i-1)}{p-1}\right\rfloor+1$.

The decomposition of $S U(n)$ is compatible with an analogous decomposition of $\Sigma \mathbb{C} P^{n-1}$. Observe that $H_{*}(S U(n)) \cong S\left(\widetilde{H}_{*}\left(\Sigma \mathbb{C} P^{n-1}\right)\right)$, and the canonical map $\Sigma \mathbb{C} P^{n-1} \longrightarrow S U(n)$ induces the inclusion of the generating set in homology. There is a decomposition [MNT1] $\Sigma \mathbb{C} P^{n-1} \simeq \bigvee_{i=1}^{p-1} A_{i}^{k}$ where $H_{*}\left(A_{i}^{k}\right)$ consists of those elements in $H_{*}\left(\Sigma \mathbb{C} P^{n-1}\right)$ in degrees $2 i+j q+1$ for values of $j \geq 0$. Specifically, $H_{*}\left(A_{i}^{k}\right)$ has vector space basis $\left\{x_{2 i+1}, x_{2 i+q+1}, \ldots, x_{2 i+(k-1) q+1}\right\}$. Observe that $H_{*}\left(\bar{B}_{i}^{k}\right) \cong S\left(\widetilde{H}_{*}\left(A_{i}^{k}\right)\right)$ and the inclusion of the generating set is realized geometrically by the composite

$$
A_{i}^{k} \longrightarrow \Sigma \mathbb{C} P^{n-1} \longrightarrow S U(n) \longrightarrow \bar{B}_{i}^{k} .
$$

This framework of homotopy decompositions and homology generation occurs for all odd-primary compact, connected, simple Lie groups $G$ which are torsion free. Here, $G$ is torsion free if its integral (co)homology has no $p$-torsion. The torsion free Lie groups are the matrix groups $\operatorname{Spin}(n), S U(n)$, and $S p(n)$ at all odd primes, and the exceptional groups $G_{2}$ at $p \geq 3, F_{4}, E_{6}$, and $E_{7}$ at $p \geq 5$, and $E_{8}$ at $p \geq 7$. The framework provides a convenient means of expressing Mimura, Nishida, and Toda's work. They did not state their results in this fashion, particularly in regard to the existence and role of the space $A(G)$ described below, so our proof of Theorem 2.1 consists mainly of identifying $A(G)$ and its wedge summands $A_{i}(G)$. We do this in two parts, first with respect to the matrix groups, and later for the exceptional groups.

Theorem 2.1. Let $G$ be a compact, connected, simple Lie group which is torsion free. Then there is a co-H space $A(G)$ such that $H_{*}(G) \cong S\left(\widetilde{H}_{*}(A(G))\right)$ and a map $A(G) \longrightarrow G$ which induces the inclusion of the generating set in homology. Further, there are homotopy decompositions

$$
A(G) \simeq \bigvee_{i=1}^{p-1} A_{i}(G) \text { and } G \simeq \prod_{i=1}^{p-1} \bar{B}_{i}(G)
$$

where $H_{*}\left(A_{i}(G)\right)$ consists of those elements in $H_{*}(A(G))$ in degrees $2 i+j q+1$ for some $j \geq 0$ and $H_{*}\left(\bar{B}_{i}(G)\right) \cong S\left(\widetilde{H}_{*}\left(A_{i}(G)\right)\right)$. Moreover, the composite

$$
A_{i}(G) \longrightarrow A(G) \longrightarrow G \longrightarrow \bar{B}_{i}(G)
$$

induces the inclusion of the generating set in homology. 
Remark 2.2. Since $G$ is finite dimensional, $H_{*}(A(G))$ and therefore $H_{*}\left(A_{i}(G)\right)$ are finite, so beyond a certain value of $j$ the elements in $H_{*}(A(G))$ in degrees $2 i+j q+1$ are all zero. Also, it may be possible that for a given $G, H_{*}\left(A_{i}(G)\right)=0$, in which case $A_{i}(G)$ is homotopy equivalent to a point.

Proof of Theorem 2.1 when $G$ is a matrix group. The argument for $G=S U(n)$ has been given: take $A(G)=\Sigma \mathbb{C} P^{n-1}$ and $A_{i}(G)=A_{i}^{k}$.

When $G=S p(n) \simeq \operatorname{Spin}(2 n+1)$, let $A(G)=\bigvee_{i=1}^{(p-1) / 2} A_{2 i-1}^{k^{\prime}}$. Note that here $A_{2 i-1}(G)=A_{2 i-1}^{k^{\prime}}$ for $1 \leq i \leq(p-1) / 2$ and $A_{2 i}(G)=*$ for $1 \leq i \leq(p-1) / 2$. Since $A(G)$ is a wedge of co- $H$ spaces, it is a co- $H$ space. Since each $\widetilde{H}_{*}\left(A_{2 i-1}^{k^{\prime}}\right)$ generates $H_{*}\left(\bar{B}_{2 i-1}^{k^{\prime}}\right)$, we have $H_{*}(G) \cong S\left(\widetilde{H}_{*}(A(G))\right)$, and adding the maps $A^{k^{\prime}} \longrightarrow \bar{B}_{i}^{k^{\prime}}$ gives a composite

$$
A(G)=\bigvee_{i=1}^{(p-1) / 2} A_{i}(G) \longrightarrow \prod_{i=1}^{(p-1) / 2} \bar{B}^{k^{\prime}} \stackrel{\simeq}{\longrightarrow} \operatorname{Sp}(n) \simeq \operatorname{Spin}(2 n+1)
$$

which induces the inclusion of the generating set in homology.

When $G=\operatorname{Spin}(2 n) \simeq S^{2 n-1} \times \operatorname{Spin}(2 n-1)$, let $A_{G}=S^{2 n-1} \vee\left(\bigvee_{i=1}^{(p-1) / 2} A_{2 i-1}^{k^{\prime \prime}}\right)$. Observe that there is a unique $i_{0}$ with $1 \leq i_{0} \leq(p-1) / 2$ such that $2 n-1=2 i_{0}+$ $t q+1$ for some $t$. If $i_{0}=2 i_{0}^{\prime}-1$, take $A_{i_{0}}(G)=A_{2 i_{0}-1}^{k^{\prime \prime}} \vee S^{2 n-1} ; A_{2 i-1}(G)=A_{2 i-1}^{k^{\prime \prime}}$ for $1 \leq i \leq(p-1) / 2$ and $i \neq i_{0}$; and $A_{2 i}(G)=*$ for $1 \leq i \leq(p-1) / 2$. If $i_{0}=2_{i_{0}}^{\prime}$, take $A_{2 i-1}(G)=A_{2 i-1}^{k^{\prime \prime}}$ for $1 \leq i \leq(p-1) / 2 ; A_{2 i_{0}^{\prime}}(G)=S^{2 n-1}$; and $A_{2 i}(G)=*$ for $1 \leq i \leq(p-1) / 2$ and $i \neq i_{0}$. The assertions of the theorem now follow as in the previous case.

Remark 2.3. The proof of Theorem 2.1 shows that when $G=\operatorname{Sp}(n)$ or $\operatorname{Spin}(2 n+1)$ then $A(G) \simeq \Sigma \mathbb{H} P^{n-1}$, the suspension of the quaternionic projective plane, and when $G=\operatorname{Spin}(2 n)$ then $A(G) \simeq S^{2 n-1} \vee \Sigma \mathbb{H} P^{n-2}$.

Mimura, Nishida, and Toda [MT, MNT2] showed that in all cases but one the torsion free exceptional Lie groups decompose as products of factors $\bar{B}_{i}^{k}$ similar to those for the matrix groups. Now, we have $H_{*}\left(\bar{B}_{i}^{k}\right) \cong \Lambda\left(x_{2 i+1}, x_{2 i+q+1}, \ldots, x_{2 i+(k-1) q+1}\right)$, but it is possible to have $i \geq p$. The action of the dual Steenrod algebra is again given by $\mathcal{P}_{*}^{j}\left(x_{2 r+1}\right)=\left(\begin{array}{l}r \\ j\end{array}\right) x_{2 r+j q+1}$. As an alternative notation, write

$$
\bar{B}_{i}^{k}=\bar{B}(2 i+1,2 i+q+1, \ldots, 2 i+(k-1) q+1) .
$$

The one special case is $G_{2}$ at the prime 3 . Here, $G_{2}$ is homotopy equivalent to a space $\bar{B}_{2}(3,11)$ with $H_{*}\left(\bar{B}_{2}(3,11)\right) \cong S\left(x_{3}, x_{11}\right)$, and the action of the dual Steenrod algebra is different from that of the spaces $\bar{B}_{i}^{k}$, as we have $\mathcal{P}_{*}^{2}\left(x_{11}\right)=x_{3}$. 
The specific homotopy decompositions are as follows:

$$
\begin{array}{lll}
G_{2} & p=3 & \bar{B} 2(3,11) \\
& p=5 & \bar{B}(3,11) \\
& p>5 & S^{3} \times S^{11} \\
F_{4} & p=5 & \bar{B}(3,11) \times \bar{B}(15,23) \\
& p=7 & \bar{B}(3,15) \times \bar{B}(11,23) \\
& p=11 & \bar{B}(3,23) \times S^{11} \times S^{15} \\
& p>11 & S^{3} \times S^{11} \times S^{15} \times S^{23} \\
E_{6} & p=5 & F_{4} \times \bar{B}(9,17) \\
& p>5 & F_{4} \times S^{9} \times S^{17} \\
E_{7} & p=5 & \bar{B}(3,11,19,27,35) \times \bar{B}(15,23) \\
& p=7 & \bar{B}(3,15,27) \times \bar{B}(11,23,35) \times S^{19} \\
& p=11 & \bar{B}(3,23) \times \bar{B}(15,35) \times S^{11} \times S^{19} \times S^{27} \\
& p=13 & \bar{B}(3,27) \times \bar{B}(11,35) \times S^{15} \times S^{19} \times S^{23} \\
& p=17 & \bar{B}(3,35) \times S^{11} \times S^{15} \times S^{19} \times S^{23} \times S^{27} \\
& p>17 & S^{3} \times S^{11} \times S^{15} \times S^{19} \times S^{23} \times S^{27} \times S^{35} \\
E_{8} & p=7 & \bar{B}(3,15,27,39) \times \bar{B}(23,35,47,59) \\
& p=11 & \bar{B}(3,23) \times \bar{B}(15,35) \times \bar{B}(27,47) \times \bar{B}(39,59) \\
& p=13 & \bar{B}(3,27) \times \bar{B}(15,39) \times \bar{B}(23,47) \times \bar{B}(35,59) \\
& p=17 & \bar{B}(3,35) \times \bar{B}(15,47) \times \bar{B}(27,59) \times S^{23} \times S^{39} \\
p=19 & \bar{B}(3,39) \times \bar{B}(23,59) \times S^{15} \times S^{27} \times S^{35} \times S^{47} \\
p=23 & \bar{B}(3,47) \times \bar{B}(15,59) \times S^{23} \times S^{27} \times S^{35} \times S^{39} \\
p=29 & \bar{B}(3,59) \times S^{15} \times S^{23} \times S^{27} \times S^{35} \times S^{39} \times S^{47} \\
p>29 & S^{3} \times S^{15} \times S^{23} \times S^{27} \times S^{35} \times S^{39} \times S^{47} \times S^{59}
\end{array}
$$

Proof of Theorem 2.1 when $G$ is an exceptional group. Observe that the product decompositions are of the form $G \simeq \prod_{i=1}^{p-1} \bar{B}_{i}(G)$ where $H_{*}\left(\bar{B}_{i}(G)\right) \cong S\left(V_{i}(G)\right)$ for a generating set $V_{i}(G)$ which consists of the generators of $H_{*}(G)$ in degrees $2 i+j q+1$ for $j \geq 0$. Note that for a given $G, V_{i}(G)$ may consist entirely of zeroes, in which case we take $\bar{B}_{i}(G)$ to be a point. We need to show there is a co- $H$ space $A_{i}(G)$ such that $H_{*}\left(A_{i}(G)\right) \cong V_{i}(G)$ and there is a map $A_{i}(G) \longrightarrow \bar{B}_{i}(G)$ which induces the inclusion of the generating set in homology. Provided we can do so, let $A(G)=\bigvee_{i=1}^{p-1} A_{i}(G)$. Then $A(G)$ is a co- $H$ space and the composite

$$
A(G) \stackrel{=}{\longrightarrow} \bigvee_{i=1}^{p-1} A_{i}(G) \longrightarrow \prod_{i=1}^{p-1} \bar{B}_{i}(G) \stackrel{\simeq}{\longrightarrow} G
$$

is a map which induces the inclusion of the generating set in homology.

To determine the spaces $A_{i}(G)$, we consider the factors $\bar{B}_{i}(G)$ one at a time. If $\bar{B}_{i}(G) \simeq *$, then let $A_{i}(G)=*$. The asserted properties of $A_{i}(G)$ and $\bar{B}_{i}(G)$ hold trivially. If $\bar{B}_{i}(G)=S^{2 m-1}$ for some $m$, then take $A_{i}(G)=S^{2 m-1}$. Observe that $A_{i}(G)$ is co- $H, H_{*}\left(\bar{B}_{i}(G)\right) \cong S\left(\widetilde{H}_{*}\left(A_{i}(G)\right)\right)$, and the identity map $A_{i}(G) \longrightarrow \bar{B}_{i}(G)$ induces the inclusion of the generating set in homology.

Suppose $\bar{B}_{i}(G)=B(2 m+1,2 m+q+1)$ for some $m$. Let $A_{i}(G)$ be the $(2 m+$ $q+1)$-skeleton of $\bar{B}_{i}(G)$. Observe that $A_{i}(G)$ has two cells in degrees $2 m+1$ and $2 m+q+1$ which in homology are connected by the dual Steenrod operation $\mathcal{P}_{*}^{1}$. So $H_{*}\left(\bar{B}_{i}(G)\right) \cong S\left(\widetilde{H}_{*}\left(A_{i}(G)\right)\right)$, as required. It remains to show that $A_{i}(G)$ is a 
co- $H$ space. The cellular description of $A_{i}(G)$ implies it is homotopy equivalent to the $(2 m-2)$-fold suspension of the homotopy cofiber of the map $S^{2 p} \stackrel{\alpha_{1}}{\longrightarrow} S^{3}$, where $\alpha_{1}$ represents a generator of $\pi_{2 p}\left(S^{3}\right) \cong \mathbb{Z} / p \mathbb{Z}$. If $2 m-2>0$, then $A_{i}(G)$ is a suspension. If $2 m-2=0$ then $A_{i}(G)$ is itself the homotopy cofiber of $\alpha_{1}$. It is well known that $\alpha_{1}$ is a co- $H$ map (it is easy to check the co- $H$ deviation vanishes) and so in all cases $A_{i}(G)$ is a co- $H$ space. The same argument applies to the special case of $G_{2} \simeq \bar{B}_{2}(3,11)$ at 3 , where $A_{i}\left(G_{2}\right)$ is now the 11 -skeleton of $\bar{B}_{2}(3,11)$.

It remains to consider the factors of rank $\geq 3$ in the decompositions of $E_{7}$ at 5 and 7 , and $E_{8}$ at 7 . All the information on the factors which follows comes from [MNT2, Section 7, Theorem 8.1]. The factor $\bar{B}(3,11,19,27,35)$ of $E_{7}$ at 5 is homotopy equivalent to the factor $\bar{B}_{1}^{5}$ of $S U(18)$. So take $A_{i}\left(E_{7}\right)=A_{1}^{5}$. Then $H_{*}(B(3,11,19,27,35)) \cong S\left(\widetilde{H}_{*}\left(A_{i}\right)\right)$ and the composite

$$
A_{i}=A_{1}^{5} \longrightarrow B_{1}^{5} \stackrel{\simeq}{\longrightarrow} \bar{B}(3,11,19,27,35)
$$

induces the inclusion of the generating set in homology. The same argument applies to the factors $\bar{B}(3,15,27)$ and $\bar{B}(11,23,35)$ of $E_{7}$ at 7 which are homotopy equivalent to the factors $\bar{B}_{1}^{3}$ and $\bar{B}_{5}^{3}$ of $S U(18)$ respectively, and the factor $\bar{B}(3,15,27,39)$ of $E_{8}$ at 7 which is homotopy equivalent to the factor $\bar{B}_{1}^{4}$ of $S U(20)$.

The factor $\bar{B}(23,35,47,59)$ of $E_{8}$ at 7 is special. It is not a retract of $S U(n)$ for any $n$. In homology, it is a retract of $H_{*}(S U(30) / S U(11))$. So to find the appropriate space $A_{5}\left(E_{8}\right)$, we look to stunted projective spaces. It follows from the decomposition of $\Sigma \mathbb{C} P^{n-1}$ in MNT1 that $\Sigma \mathbb{C} P^{29} / \Sigma \mathbb{C} P^{10} \simeq \bigvee_{i=1}^{6} A_{i}$ where $\widetilde{H}_{*}\left(A_{i}\right)$ consists of those elements in $H_{*}\left(\Sigma \mathbb{C} P^{29} / \Sigma \mathbb{C} P^{10}\right)$ in degrees $2 i+j q+1$ for $j \geq 0$. In particular, $A_{5}$ has vector space basis $\left\{x_{23}, x_{35}, x_{47}, x_{59}\right\}$, and the action of the dual Steenrod algebra is such that $H_{*}(\bar{B}(23,35,47,59)) \cong S\left(\widetilde{H}_{*}\left(A_{5}\right)\right)$. Note that $A_{5}$ is a co- $H$ space because it retracts off a co- $H$ space. Taking $A_{5}\left(E_{7}\right)=A_{5}$, to finish the proof we need to find a map $A_{5} \longrightarrow \bar{B}(23,35,47,59)$ which realizes the inclusion of the generating set in homology. Such a map can be constructed by iteratively extending the inclusion of the bottom cell $S^{23} \longrightarrow \bar{B}(23,35,47,59)$ to the 35,47 , and 59 skeletons of $A_{5}$. The obstructions to doing so lie in $\pi_{t}(B(23,35,47,59))$ for $t \in\{34,46,58\}$. But all such groups can be seen to be zero by applying the homotopy spectral sequence of [MNT2, Section 6].

As a final remark, observe that the condition $H_{*}\left(\bar{B}_{i}(G)\right) \cong S\left(\widetilde{H}_{*}\left(A_{i}(G)\right)\right)$ in Theorem 2.1 suggests that $\bar{B}_{i}(G)$ may be universal for $A_{i}(G)$. But while $\bar{B}_{i}(G)$ is an $H$-space because it retracts off $G$, the construction of $\bar{B}_{i}(G)$ does not lend itself well to determining whether or not it is homotopy associative and homotopy commutative. The universal space for $A_{i}(G)$, provided $n \leq(p-1)(p-3)$, will be obtained by a different construction described in Section 4 .

\section{UNIVERSALITY}

This is a preliminary section in which we expand upon the notion of universality discussed in the Introduction, and prove three lemmas to be used later on. The universal property we are interested in pertains to homotopy associative, homotopy commutative $H$-spaces. The analogue for $H$-spaces which are only homotopy associative is much more familiar; it is the James construction, so we begin with it. 
Let $X$ be a connected space. Let $X^{(i)}$ be the $i$-fold smash product of $X$ with itself. Let $E: X \longrightarrow \Omega \Sigma X$ be the suspension map. Let $J(X)$ be the free monoid on the points of $X$, with the basepoint as the identity. James [J] proved:

Theorem 3.1. The following hold:

(a) there is a homotopy equivalence $J(X) \simeq \Omega \Sigma X$,

(b) there is a homotopy equivalence $\Sigma \Omega \Sigma X \simeq \bigvee_{i=1}^{\infty} \Sigma X^{(i)}$,

(c) if $Y$ is a homotopy associative $H$-space and there is a map

$f: X \longrightarrow Y$, then there is a unique (up to homotopy) H-map

$\bar{f}: \Omega \Sigma X \longrightarrow Y$ with the property that $\bar{f} \circ E \simeq f$.

The universal property is given by item (c) in Theorem 3.1. In particular, it implies there is a one-to-one correspondence between the set of homotopy classes of maps from $X$ to $Y$ and the set of homotopy classes of $H$-maps from $\Omega \Sigma X$ to $Y$.

Now we turn to homotopy associative, homotopy commutative $H$-spaces. Given a space $A$, we would like to find a homotopy associative, homotopy commutative $H$ space $B$ which is universal for it, in the sense that any map from $A$ to a homotopy associative, homotopy commutative $H$-space $Z$ extends uniquely to an $H$-map from $B$ to $Z$. To be precise, we make the following definition.

Definition 3.2. Let $A$ be a space. A homotopy associative, homotopy commutative $H$-space $B$ is universal for $A$ if there is a map $i: A \longrightarrow B$ (which is to be regarded as an inclusion) with the following property: given a map $f: A \longrightarrow Z$ where $Z$ is homotopy associative and homotopy commtutative, then there is a unique $H$-map $\bar{f}: B \longrightarrow Z$ such that $\bar{f} \circ i \simeq f$.

There is no known functorial construction of such a space $B$. The known examples of universal spaces have all been $a d$ hoc constructions. A basic example which will be referred to later is the following.

Example 3.3. If $p \geq 5$, then $S^{2 n+1}$ is its own universal space. It is homotopy associative and homotopy commutative. Take $i: S^{2 n+1} \longrightarrow S^{2 n+1}$ to be the identity map. Then Gray [G] showed that any map from $S^{2 n+1}$ to $S^{2 m+1}$ is homotopic to an $H$-map. His method in fact shows that any map from $S^{2 n+1}$ to a homotopy commutative, homotopy associative $H$-space $Z$ is homotopic to an $H$-map.

Note that if a space $A$ has a universal space, then it is unique. For if $B$ and $B^{\prime}$ are both universal for $A$, then the inclusions $A \stackrel{i}{\longrightarrow} B$ and $A \stackrel{i^{\prime}}{\longrightarrow} B^{\prime}$ can be extended to give $H$-maps $B^{\prime} \stackrel{\bar{i}}{\longrightarrow} B$ and $B \stackrel{\bar{i}^{\prime}}{\longrightarrow} B^{\prime}$. The identity map $1_{B}$ on $B$ and $\bar{i} \circ \bar{i}^{\prime}$ are both $H$-maps which extend $A \stackrel{i}{\longrightarrow} B$, so the uniqueness property of the multiplicative extension implies that $\bar{i} \circ \bar{i}^{\prime} \simeq 1_{B}$. Similarly, $\bar{i}^{\prime} \circ \bar{i} \simeq 1_{B^{\prime}}$. Thus there is an $H$-equivalence $B \simeq B^{\prime}$.

The value of this universal property comes from the fact that the uniqueness of the multiplicative extensions implies there is a one-to-one correspondence between homotopy classes of maps from $A$ to $Z$ and homotopy classes of $H$-maps from $B$ to $Z$. One common use of this in practice is to show that two $H$-maps $B \longrightarrow Z$ are homotopic by showing that their restrictions to $A$ are homotopic. Doing exactly this is the key point behind the proofs of the following three lemmas, which will be used subsequently. 
Lemma 3.4. Suppose $B_{1}$ and $B_{2}$ are homotopy associative, homotopy commutative $H$-spaces which are universal for spaces $A_{1}$ and $A_{2}$. Then $B_{1} \times B_{2}$ is a homotopy associative, homotopy commutative $H$-space which is universal for $A_{1} \vee A_{2}$.

Proof. The product $H$-structure on $B_{1} \times B_{2}$ is clearly homotopy associative and homotopy commutative. By hypothesis, there are inclusions $i_{1}: A_{1} \longrightarrow B_{1}$ and $i_{2}: A_{2} \longrightarrow B_{2}$. Let $i$ be the composite

$$
i: A_{1} \vee A_{2} \stackrel{i_{1} \vee i_{2}}{\longrightarrow} B_{1} \vee B_{2} \stackrel{j}{\longrightarrow} B_{1} \times B_{2}
$$

where $j$ is the inclusion of the wedge into the product. Let $Z$ be a homotopy associative, homotopy commutative $H$-space and suppose there is a map $f: A_{1} \vee$ $A_{2} \longrightarrow Z$. We need to show that there is a unique $H$-map $\bar{f}: B_{1} \times B_{2} \longrightarrow Z$ such that $\bar{f} \circ i \simeq f$.

We first show existence. Since the domain of $f$ is a wedge, it is determined by its restrictions $f_{1}$ and $f_{2}$ to $A_{1}$ and $A_{2}$ respectively. Since $B_{1}$ and $B_{2}$ are universal for $A_{1}$ and $A_{2}$, there exist unique $H$-maps $\bar{f}_{1}: B_{1} \longrightarrow Z$ and $\bar{f}_{2}: B_{2} \longrightarrow Z$ such that $\bar{f}_{1} \circ i_{1} \simeq f_{1}$ and $\bar{f}_{2} \circ i_{2} \simeq f_{2}$. Let $\bar{f}$ be the composite

$$
\bar{f}: B_{1} \times B_{2} \stackrel{\bar{f}_{1} \times \bar{f}_{2}}{\longrightarrow} Z \times Z \stackrel{\mu}{\longrightarrow} Z
$$

where $\mu$ is the multiplication on $Z$. Since $Z$ is homotopy associative and homotopy commutative, $\mu$ is an $H$-map. Thus $\bar{f}$ is a composite of $H$-maps and so is an $H$-map. Now consider the homotopy commutative diagram

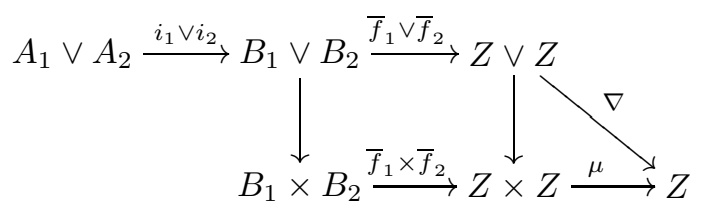

where $\nabla$ is the folding map. The lower direction around the diagram is the definition of $\bar{f} \circ i$. The top row is $\bar{f}_{1} \circ i_{1} \vee \bar{f}_{2} \circ i_{2} \simeq f_{1} \vee f_{2}$. Thus the upper direction around the diagram is homotopic to $f$. Hence $\bar{f} \circ i \simeq f$.

To show uniqueness, suppose $g: B_{1} \times B_{2} \longrightarrow Z$ is another $H$-map such that $g \circ i \simeq f$. For $t=1$ or $t=2$ let $g_{t}$ be the composite

$$
g_{t}: B_{t} \longrightarrow B_{1} \times B_{2} \stackrel{g}{\longrightarrow} Z
$$

where the left map is the inclusion into the $t^{\text {th }}$-wedge summand. Observe that the inclusion $B_{t} \longrightarrow B_{1} \times B_{2}$ is an $H$-map, so $g_{t}$ is an $H$-map, as it is the composite of $H$-maps. Since $Z$ is homotopy associative and homotopy commutative, $g$ is homotopic to the sum of its restrictions, that is, to the composite

$$
B_{1} \times B_{2} \stackrel{g_{1} \times g_{2}}{\longrightarrow} Z \times Z \stackrel{\mu}{\longrightarrow} Z .
$$

Thus $f \simeq g \circ i \simeq \mu \circ\left(g_{1} \times g_{2}\right) \circ j \circ\left(i_{1} \vee i_{2}\right)$. Restricting to $i_{t}$ shows that $g_{t} \circ i_{t} \simeq f_{t}$. The universal property of $B_{t}$ for $A_{t}$ then implies that $g_{t} \simeq \bar{f}_{t}$. Thus $g \simeq \mu \circ\left(g_{1} \times g_{2}\right) \simeq$ $\mu \circ\left(\bar{f}_{1} \times \bar{f}_{2}\right) \simeq f$.

If $A$ is a co- $H$ space, let $\underline{p}: A \longrightarrow A$ be the degree $p$ map. If $B$ is an $H$-space, let $p: B \longrightarrow B$ be the $p^{t h}$-power map. Note that if $B$ is homotopy associative and homotopy commutative, then $p$ is an $H$-map. An odd dimensional sphere, $S^{2 n+1}$, is both a co- $H$ space and an $H$-space, and the degree $p$ map is homotopic to the $p^{\text {th }}$-power map; both maps will be commonly denoted by $p$. 
Lemma 3.5. Suppose $B$ is a homotopy associative, homotopy commutative $H$ space which is universal for a co-H space $A$. Then there is a homotopy commutative diagram

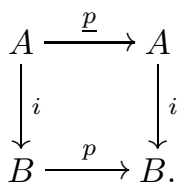

Proof. We begin with an observation about looped Whitehead products. Let $w_{j}: \Sigma A^{(j)} \longrightarrow \Sigma A$ be the $j$-fold iterated Whitehead product of the identity map on $\Sigma A$ with itself. The adjoint of $w_{j}$ is a Samelson product, and is homotopic to the composite $A^{(j)} \stackrel{E}{\longrightarrow} \Omega \Sigma A^{(j)} \stackrel{\Omega w_{j}}{\longrightarrow} \Omega \Sigma A$. The James construction extends $A \stackrel{i}{\longrightarrow} B$ to an $H$-map $r: \Omega \Sigma A \longrightarrow B$. As $B$ is homotopy associative and $r$ is an $H$-map, the composite $r \circ\left(\Omega w_{j} \circ E\right)$ is a Samelson product, and as $B$ is homotopy commutative, this Samelson product is null homotopic. The uniqueness assertion of the James construction says that the $H$-map $r \circ \Omega w_{j}$ is determined by its restriction to $A$ by $E$. Thus the triviality of $r \circ \Omega w_{j} \circ E$ implies that $r \circ \Omega w_{j}$ is null homotopic.

Returning to the main problem, as in [C, §4], the Hilton-Milnor theorem shows that the difference $\Omega \Sigma A \stackrel{p-\Omega \Sigma p}{\longrightarrow} \Omega \Sigma A$ is homotopic to a sum $\Sigma_{\alpha}\left(\Omega w_{\alpha} \circ H_{\alpha}\right)$, where $H_{\alpha}$ is a Hilton-Hopf invariant and $w_{\alpha}$ is an iterated Whitehead product of the identity map on $\Sigma A$ with itself. Composing with the $H$-map $\Omega \Sigma A \stackrel{r}{\longrightarrow} B$ lets us distribute on the left, giving $r \circ(p-\Omega \Sigma \underline{p}) \simeq r \circ\left(\Sigma_{\alpha}\left(\Omega w_{\alpha} \circ H_{\alpha}\right)\right) \simeq \Sigma_{\alpha}\left(r \circ \Omega w_{\alpha} \circ H_{\alpha}\right)$. Since $r$ is an $H$-map and $B$ is homotopy associative and homotopy commutative, the argument in the first paragraph shows that $r \circ \Omega w_{\alpha}$ is null homotopic for each $\alpha$. Thus $r \circ(p-\Omega \Sigma \underline{p}) \simeq *$ and so $r \circ p \simeq r \circ \Omega \Sigma \underline{p}$. Since $r$ is an $H$-map, $r \circ p \simeq p \circ r$, and so $p \circ r \simeq r \circ \Omega \Sigma p$. This proves the homotopy commutativity of the right square in the following diagram:

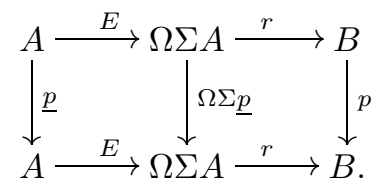

The left square homotopy commutes by the naturality of $E$. The definition of $r$ as an extension of $i$ via the James construction implies that $i \simeq r \circ E$. Thus the homotopy commutativity of the outer rectangle shows that $p \circ i \simeq i \circ \underline{p}$, proving the lemma.

Lemma 3.6. Let $X_{1} \longrightarrow X_{2} \longrightarrow X_{3}$ be a homotopy cofibration of path-connected spaces. Suppose for each $X_{i}$ there is a homotopy associative, homotopy commutative $H$-space $Y_{i}$ which is universal for $X_{i}$, and which has the property that $H_{*}\left(Y_{i}\right) \cong$ $S\left(\widetilde{H}_{*}\left(X_{i}\right)\right)$. Suppose in addition that $\widetilde{H}_{*}\left(X_{2}\right) \cong \widetilde{H}_{*}\left(X_{1}\right) \oplus \widetilde{H}_{*}\left(X_{3}\right)$. Then there are $H$-maps $Y_{1} \rightarrow Y_{2}, Y_{2} \rightarrow Y_{3}$ and a homotopy commutative diagram

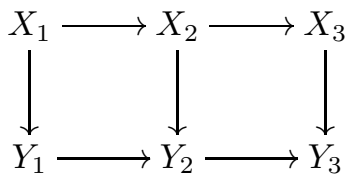

in which the bottom row is a multiplicative homotopy fibration. 
Proof. For $1 \leq j \leq 3$, let $i_{j}: X_{j} \longrightarrow Y_{j}$ be the inclusion into the universal space. The universal property of $Y_{1}$ for $X_{1}$ implies there is an $H$-map $g_{1}: Y_{1} \longrightarrow Y_{2}$ extending the composite $X_{1} \longrightarrow X_{2} \stackrel{i_{2}}{\longrightarrow} Y_{2}$. Similarly, there is an $H$-map $g_{2}: Y_{2} \longrightarrow Y_{3}$ extending the composite $X_{2} \longrightarrow X_{3} \stackrel{i_{3}}{\longrightarrow} Y_{3}$. This proves the homotopy commutativity of the asserted diagram. Since the composite $\theta: X_{1} \longrightarrow X_{2} \longrightarrow X_{3}$ is null homotopic, both $g_{2} \circ g_{1}$ and the trivial map are $H$-maps extending the composite $X_{1} \stackrel{\theta}{\longrightarrow} X_{3} \stackrel{i_{3}}{\longrightarrow} Y_{3}$. The universal property says that such a multiplicative extension is unique, and so $g_{2} \circ g_{1}$ is null homotopic.

It remains to show that the bottom row of the diagram in the statement of the lemma is a homotopy fibration. Let $F$ be the homotopy fiber of $Y_{2} \stackrel{g_{2}}{\longrightarrow} Y_{3}$. Since $g_{2}$ is an $H$-map, $F$ is an $H$-space and the fiber map $h: F \longrightarrow Y_{2}$ is an $H$ map. The Serre spectral sequence converging to $H_{*}(F)$ is therefore multiplicative. The homological hypotheses imply that this spectral sequence collapses at the $E_{2}$ term, $H_{*}(F) \cong S\left(\widetilde{H}_{*}\left(X_{1}\right)\right)$, and $h_{*}$ is the subalgebra inclusion $S\left(\widetilde{H}_{*}\left(X_{1}\right)\right) \longrightarrow$ $S\left(\widetilde{H}_{*}\left(X_{1}\right) \oplus \widetilde{H}_{*}\left(X_{2}\right)\right)$. Note that $h_{*}=\left(g_{1}\right)_{*}$. The null homotopy $g_{2} \circ g_{1} \simeq *$ implies that $g_{2}$ lifts to a map $\lambda: Y_{1} \longrightarrow F$. Since $h_{*}=\left(g_{1}\right)_{*}$ and both are injective, $\lambda_{*}$ must be an isomorphism. Since $Y_{1}$ and $F$ are path-connected, Whitehead's theorem says that $\lambda$ is a homotopy equivalence. It follows that the sequence $Y_{1} \stackrel{g_{1}}{\longrightarrow} Y_{2} \stackrel{g_{2}}{\longrightarrow} Y_{3}$ is a homotopy fibration.

\section{Alternative Low Rank Decompositions of Lie groups}

The purpose of this section is to prove the decompositions in Theorem 1.1. The factors in these new decompositions arise from Cohen and Neisendorfer's [CN] construction of low rank, torsion free, finite $H$-spaces, stated in Theorem 4.1. Conditions describing when they are homotopy associative, homotopy commutative, and universal were given by the author in $[\mathrm{T}$, and are stated in Theorem 4.2 .

Theorem 4.1. Let $X$ be a $C W$-complex consisting of $l$ odd dimensional cells, where $l<p-1$. Then there is an $H$-space $Y$ such that $H_{*}(Y) \cong S\left(\widetilde{H}_{*}(X)\right)$ and there is a map $X \longrightarrow Y$ which induces the inclusion of the generating set in homology.

Theorem 4.2. Given the hypotheses in Theorem 4.1, suppose in addition that $X$ is a suspension and $l<p-2$. Then the corresponding $H$-space $Y$ is homotopy associative, homotopy commutative, and universal for $X$.

The additional hypothesis that $X$ is a suspension in Theorem 4.2 is a little too strong for the application we have in mind. What is needed is a statement which is valid when $X$ is a co- $H$ space. We prove this in the following proposition.

Proposition 4.3. Given the hypotheses in Theorem 4.1, suppose in addition that $X$ is a co-H space and $l<p-2$. Then the corresponding $H$-space $Y$ is homotopy associative, homotopy commutative, and universal for $X$.

Proof. Examining the proof of Theorem 4.2 (which appears as Theorem $1.1 \mathrm{in}$ [T]), there was only one instance in which the hypothesis that $X$ is a suspension was used. This occurred in Lemma 2.2 of that paper and arose as follows. Let $a d^{1}$ : $X \longrightarrow \Omega \Sigma X$ be the suspension map. For $k \geq 2$, let

$$
a d^{k}: X^{(k)} \longrightarrow \Omega \Sigma X
$$

be the $k$-fold iterated Samelson product defined recursively by $a d^{k}=\left[E, a d^{k-1}\right]$. Suppose $X$ is a suspension. Then $X^{(k)}$ for $k \geq 2$ is a double suspension and so the 
set of homotopy classes of maps $\left[X^{(k)}, X^{(k)}\right]$ has the structure of an abelian group. Observe that if $\sigma$ is a permutation in the symmetric group $\Sigma_{k}$ on $k$ letters, then there is a corresponding map $\sigma: X^{(k)} \longrightarrow X^{(k)}$ defined by permuting the smash factors. Define a map

$$
\beta_{k}: X^{(k)} \longrightarrow X^{(k)}
$$

inductively by letting $\beta_{2}=1-(1,2)$ and $\beta_{k}=(1-(k, k-1, \ldots, 2,1)) \circ\left(1 \wedge \beta_{k-1}\right)$. In homology, $\left(\beta_{k}\right)_{*}\left(x_{1} \otimes \cdots \otimes x_{k}\right)=\left[x_{1},\left[x_{2}, \ldots\left[x_{k-1}, x_{k}\right] \ldots\right]\right]$. The abelian group structure on $\left[X^{(k)}, X^{(k)}\right]$ lets us carry out the same arithmetic that Dynkin, Specht, and Wever $[\mathrm{Sp}, \mathrm{W}]$ used on the group ring $\mathbb{Z}_{(p)}\left[\Sigma_{k}\right]$ to show that $\beta_{k} \circ \beta_{k} \simeq k \cdot \beta_{k}$. Thus if we restrict to $k<p$ and define $\bar{\beta}_{k}=\frac{1}{k} \beta_{k}$, then $\bar{\beta}_{k}$ is an idempotent. Let $Q_{k}=\operatorname{hocolim}_{\bar{\beta}_{k}} X^{(k)}$. The linearity of the Samelson product then implies that there is a homotopy commutative diagram

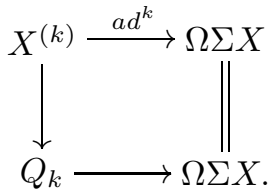

The existence of this homotopy commutative diagram is the content of Lemma 2.2 of $[\mathrm{T}$.

Now weaken the hypothesis that $X$ is a suspension to $X$ being a co- $H$ space. The set $\left[X^{(k)}, X^{(k)}\right]$ only has the structure of a group, but $\Sigma X$ is homotopy cocommutative so $\left[\Sigma X^{(k)}, \Sigma X^{(k)}\right]$ has the structure of an abelian group. We can therefore similarly define a map $\bar{\beta}_{k}: \Sigma X^{(k)} \longrightarrow \Sigma X^{(k)}$ as before and $\bar{\beta}_{k}=\frac{1}{k} \beta_{k}$ is an idempotent if $k<p$. Let $\bar{Q}_{k}=\operatorname{hocolim}_{\bar{\beta}_{k}} \Sigma X^{(k)}$. The adjoint of $a d^{k}$ is the $k$-fold iterated Whitehead product $w_{k}: \Sigma X^{(k)} \longrightarrow \Sigma X$ of the identity map on $\Sigma X$ with itself. The bilinearity of the Whitehead product can then be used in place of the bilinearity of the Samelson product to show there is a homotopy commutative diagram

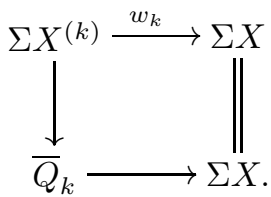

As the arguments in [T] are phrased in terms of Whitehead products rather than Samelson products, it was really the adjoint of the factorization of $a d^{k}$ through $Q_{k}$ that was used. That is, the factorization of $w_{k}$ through $\bar{Q}_{k}$ would have sufficed to carry the argument through. As this factorization also holds when $X$ is a co- $H$ space, the proof of Theorem 4.2 is equally valid in that case too.

We now use Theorem 4.1 and Proposition 4.3 to produce the spaces which will be the factors appearing in the decompositions of low rank Lie groups in Theorem 1.1 For each compact, connected, simple Lie group $G$ which is torsion free, Theorem 2.1 shows that there is a co- $H$ space $A(G)$ such that $H_{*}(G) \cong S\left(\widetilde{H}_{*}(A(G))\right)$ and there is a map $A(G) \longrightarrow G$ which induces the inclusion of the generating set in homology. The space $A(G)$ decomposes as $A(G) \simeq \bigvee_{i=1}^{p-1} A_{i}(G)$, where $H_{*}\left(A_{i}(G)\right)$ consists of those elements in $H_{*}(A(G))$ in degrees $2 i+j q+1$ for $j \geq 0$. Observe that $A_{i}(G)$ is a co- $H$ space because it retracts off a co- $H$ space. Restricting to the groups $G$ 
listed in Theorem 1.1 we see that each of the $p-1$ wedge summands $A_{i}(G)$ has at most $p-3$ cells, and all are in odd dimensions. Theorem 4.1 and Proposition 4.3 then apply to show:

Lemma 4.4. There exists a homotopy associative, homotopy commutative $H$-space $B_{i}(G)$ with $H_{*}\left(B_{i}(G)\right) \cong S\left(\widetilde{H}_{*}\left(A_{i}(G)\right)\right)$ and $B_{i}(G)$ is universal for $A_{i}(G)$.

Recall the factors $\bar{B}_{i}(G)$ in the decomposition of $G$ in Section 2. Observe that there are abstract isomorphisms $H_{*}\left(\bar{B}_{i}(G)\right) \cong S\left(\widetilde{H}_{*}\left(A_{i}(G)\right)\right) \cong H_{*}\left(B_{i}(G)\right)$. We wish to realize these ismorphisms geometrically by showing that $\bar{B}_{i}(G)$ and $B_{i}(G)$ are homotopy equivalent. To do so, we first prove a general lemma.

Lemma 4.5. Let $A$ be a space consisting of $l$ odd dimensional cells, where $l<p$. Suppose: (i) there are $H$-spaces $B_{1}$ and $B_{2}$ satisfying coalgebra isomorphisms $H_{*}\left(B_{1}\right) \cong S\left(\widetilde{H}_{*}(A)\right) \cong H_{*}\left(B_{2}\right)$, and (ii) there are maps $f_{1}: A \longrightarrow B_{1}$ and $f_{2}: A \longrightarrow B_{2}$ which induce the inclusion of the generating set in homology. Then there is a homotopy equivalence $B_{1} \simeq B_{2}$.

Proof. Let $j \in\{1,2\}$. Since $B_{j}, j=1$ or 2 , is an $H$-space, the James construction gives a map $\bar{f}_{j}: \Omega \Sigma A \longrightarrow B_{j}$ such that $\bar{f}_{j} \circ E \simeq f_{j}$. Note that the James construction is usually applied when the target is a homotopy associative $H$-space, in which case the extension $\bar{f}_{j}$ would be an $H$-map. In our case, $B_{j}$ is an $H$ space but not homotopy associative. However, the James construction can still be applied, but the extension $\bar{f}_{j}$ need not be an $H$-map. The upshot is that in homology we have a coalgebra map $T\left(\widetilde{H}_{*}(A)\right) \stackrel{\left(\bar{f}_{j}\right)_{*}}{\longrightarrow} S\left(\widetilde{H}_{*}(A)\right)$, and the homotopy $\bar{f}_{j} \circ E \simeq f_{j}$ implies that $\left(\bar{f}_{j}\right)_{*}$ sends the generating set of $T\left(\widetilde{H}_{*}(A)\right)$ isomorphically onto the generating set of $S\left(\widetilde{H}_{*}(A)\right)$.

As in [CN 4.1], the condition that the number $l$ of odd dimensional cells in $A$ is less than $p$ implies that $\bar{f}_{j}$ has a right homotopy inverse $s_{j}: B_{j} \longrightarrow \Omega \Sigma A$. Consider the composite

$$
g: B_{1} \stackrel{s_{1}}{\longrightarrow} \Omega \Sigma A \stackrel{\bar{f}_{2}}{\longrightarrow} B_{2}
$$

It is more convenient to use cohomology at this point. Observe that each element in $\widetilde{H}_{*}(A)$ is of odd degree so $S\left(\widetilde{H}_{*}(A)\right)$ is an exterior algebra, so it is therefore isomorphic to its dual. Let $V$ be the dual of $\widetilde{H}_{*}(A)$ so $H^{*}\left(B_{j}\right) \cong S(V)$. Dualizing the last sentence of the previous paragraph shows that if $x \in V$, then $\left(\bar{f}_{1}\right)^{*}(x)=$ $\left(\bar{f}_{2}\right)^{*}(x)$. Since $s_{1}$ is a right homotopy inverse of $\bar{f}_{1}$, we have $\left(s_{1}\right)^{*} \circ\left(\bar{f}_{1}\right)^{*}(x)=x$. Thus $\left(s_{1}\right)^{*} \circ\left(\bar{f}_{2}\right)^{*}(x)=x$. That is, $g^{*}$ is an isomorphism on algebra generators. Hence $g^{*}$ is an isomorphism and so $g$ is a homotopy equivalence.

Proof of Theorem 1.1. Let $G$ be one of the Lie groups listed in the hypotheses. In Theorem 2.1 we saw that there is a space $A(G)$ and a map $A(G) \longrightarrow G$ such that $H_{*}(G) \cong S\left(\widetilde{H}_{*}(A(G))\right)$. Further, there are homotopy decompositions $G \simeq$ $\prod_{i=1}^{p-1} \bar{B}_{i}(G)$ and $A(G) \simeq \bigvee_{i=1}^{p-1} A_{i}(G)$ such that $H_{*}\left(\bar{B}_{i}(G)\right) \cong S\left(\widetilde{H}_{*}\left(A_{i}(G)\right)\right)$ and the composite

$$
A_{i}(G) \longrightarrow A(G) \longrightarrow G \longrightarrow \bar{B}_{i}(G)
$$

induces the inclusion of the generating set in homology.

On the other hand, Lemma 4.4 shows that the universal space $B_{i}(G)$ of $A_{i}(G)$ also has the property that $H_{*}\left(B_{i}(G)\right) \cong S\left(\widetilde{H}_{*}\left(A_{i}(G)\right)\right)$ and the map $A_{i}(G) \longrightarrow$ 
$B_{i}(G)$ induces the inclusion of the generating set in homology. Lemma 4.5 then shows that $\bar{B}_{i}(G)$ and $B_{i}(G)$ are homotopy equivalent. Hence $G \simeq \prod_{i=1}^{p-1} B_{i}(G)$, completing the proof.

Remark 4.6. Observe that the list of Lie groups $G$ to which Theorem 1.1 applies excludes the torsion free cases of $G_{2}$ at 3 and $E_{7}$ at 5 . This is due to the restriction that the number of cells of $X$ is less than or equal to $p-3$, which arises from Proposition 4.3. In particular, the theorem never applies to a nontrivial $X$ when $p=3$, and does not apply to the factor $\bar{B}(3,11,19,27,35)$ of $E_{7}$, as its corresponding space " $X$ " has 5 cells.

\section{EXPONENTS}

In this section we apply the universal properties to prove the factorization in Theorem 1.3, which leads to the exponent result in Theorem 1.5. We do so by proving a more general statement, Theorem 5.1. Basically, the suspension of a complex projective space or a stunted complex projective space has a canonical decomposition into a wedge of $p-1$ summands. If $A$ is one of the summands, and if it has at most $p-3$ cells, then there is a homotopy associative, homotopy commutative $H$-space $B$ which is universal for $A$. Moreover, $B$ is spherically resolved. We use information about the action of the Steenrod algebra on $H_{*}(A)$ to prove that multiplication by $p^{r}$ on $B$ - for a suitably small $r$ - factors through the product of the resolving spheres of $B$.

In what follows, we are going to consider co- $H$ spaces $A_{i}^{k}$ such that there is a vector space isomorphism

$$
\widetilde{H}_{*}\left(A_{i}^{k}\right) \cong\left\{x_{2 i+1}, x_{2 i+q+1}, \ldots, x_{2 i+(k-1) q+1}\right\}
$$

and the action of the Steenrod algebra is given by $\mathcal{P}^{j}\left(x_{2 r+1}\right)=\left(\begin{array}{l}r \\ j\end{array}\right) x_{2 r+j q+1}$. Note that $k$ records the number of cells in $A_{i}^{k}$. Let $\mathcal{C}$ be the collection of all such spaces. If $A_{i}^{k} \in \mathcal{C}$, then by Theorem 4.1 and Proposition 4.3 there is a homotopy associative, homotopy commutative $H$-space $B_{i}^{k}$ which is universal for $A_{i}^{k}$. Note that as all generators in $\widetilde{H}_{*}\left(A_{i}^{k}\right)$ are of odd degree, the symmetric algebra $S\left(\widetilde{H}_{*}\left(A_{i}^{k}\right)\right)$ is an exterior algebra and so is isomorphic to its dual. Thus, by Theorem 4.1. $H^{*}\left(B_{i}^{k}\right) \cong$ $S\left(\widetilde{H}^{*}\left(A_{i}^{k}\right)\right)$. So $k$ records the rank of $B_{i}^{k}$.

Spaces $A_{i}^{k}$ always exist for each $1 \leq i, k<\infty$. They can be realized as retracts of suspensions of stunted complex projective spaces. Let $\mathbb{C} P_{m}^{n}=\mathbb{C} P^{n} / \mathbb{C} P^{m-1}$ be the stunted projective space. Note that when $m=1$ we have $\mathbb{C} P_{1}^{n}=\mathbb{C} P^{n}$, so we are considering the complex projective spaces and the (genuinely) stunted projective spaces simultaneously. A corollary of the homotopy decomposition of $\Sigma \mathbb{C} P^{n}$ discussed in Section 2 is a homotopy decomposition

$$
\Sigma \mathbb{C} P_{m}^{n} \simeq \bigvee_{j=1}^{p-1} A_{j}
$$

where $\widetilde{H}^{*}\left(A_{j}\right)$ has vector space basis $\left\{x_{2 i+1}, x_{2 i+q+1}, \ldots, x_{2 i+(k-1) q+1}\right\}$ for $i=$ $m+j-1$ and $k=\left\lfloor\frac{n-i}{p-1}\right\rfloor+1$, and the action of the Steenrod algebra is given by $\mathcal{P}^{s}\left(x_{2 r+1}\right)=\left(\begin{array}{l}r \\ s\end{array}\right) x_{2 r+s q+1}$. So $A_{j}$ is a realization of $A_{i}^{k}$.

Fix $i$ and $k$. We wish to record the number of generators in $\widetilde{H}^{*}\left(A_{i}^{k}\right)$ on which the Steenrod operation $\mathcal{P}^{1}$ acts nontrivially. Let $c_{i}^{k}$ be the number of nonzero elements 
in the set $\left\{\mathcal{P}^{1}\left(x_{2 i+1}\right), \ldots, \mathcal{P}^{1}\left(x_{2 i+(k-2) q+1}\right)\right\}$. Here, $x_{2 i+(k-1) q+1}$ has been excluded as $\mathcal{P}^{1}$ acts trivially on it for dimensional reasons. Observe that if $1 \leq t<p-1$, then

$$
\mathcal{P}^{1}\left(x_{2 i+(t-1) q+1}\right)=\left(\begin{array}{c}
i+(t-1)(p-1) \\
1
\end{array}\right) x_{2 i+t q+1}=(i+(t-1)(p-1)) x_{2 i+t q+1} .
$$

As $1 \leq t<p-1$, we have $i+(t-1)(p-1) \cong 0(\bmod p)$ for exactly one value of $t$. Thus there is at most one instance where consecutive generators of $\widetilde{H}^{*}\left(A_{i}^{k}\right)$ are not connected by $\mathcal{P}^{1}$. So if every generator $x_{2 r+1}$ of $\widetilde{H}^{*}\left(A_{i}^{k}\right)$ satisfies $\mathcal{P}^{1} x_{2 r+1}=$ $u \cdot x_{2 r+q+1}$ where $u \neq 0$, then $c_{i}^{k}=k-1$; otherwise, $c_{i}^{k}=k-2$.

In what follows, we will need to make certain statements about the degrees of maps to and from spheres. These will often be multiples of $p$, in which case they are zero in mod- $p$ cohomology. So for the degree statements alone, we use $\mathbb{Z}_{(p)^{-}}$ cohomology and for all other statements we use mod- $p$ cohomology. Observe that no real discrepancies arise between the two in our case since the spaces $A_{i}^{k}$ are retracts of $\mathbb{C} P^{n}$ for some $n$, and so $\widetilde{H}_{*}\left(A_{i}^{k} ; \mathbb{Z} / p \mathbb{Z}\right) \cong \widetilde{H}_{*}\left(A_{i}^{k} ; \mathbb{Z}_{(p)}\right) \otimes \mathbb{Z} / p \mathbb{Z}$. We continue to use $H^{*}($ ) to mean mod-p cohomology.

Theorem 5.1. Let $1 \leq k \leq p-3$. Then there is a homotopy commutative diagram of $H$-spaces and $H$-maps

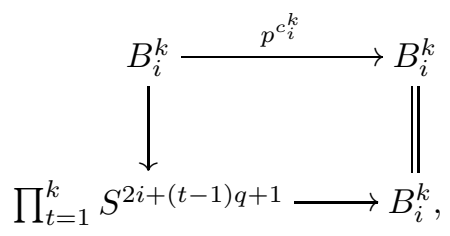

where the composite $S^{2 i+(t-1) q+1} \hookrightarrow \prod_{i=1}^{k} S^{2 i+(t-1) q+1} \longrightarrow B_{i}^{k}$ has degree $p^{c_{i}^{t}}$ in $\mathbb{Z}_{(p)}$-cohomology and the composite $B_{i}^{k} \longrightarrow \prod_{t=1}^{p-1} S^{2 i+(t-1) q+1} \rightarrow S^{2 i+(t-1) q+1}$ has degree $p^{c_{i}^{k}-c_{i}^{t}}$ in $\mathbb{Z}_{(p)}$-cohomology.

Remark 5.2. It should be emphasized that Theorem 5.1 has two useful features. It may be the case that it can be shown for other more general reasons that some power of $p$ on $B_{i}^{k}$ factors through the product of the resolving spheres. Theorem 5.1 gives $c_{i}^{k}$ as a precise power of $p$ for which this works, and it makes explicit what the maps are into and out of the product of spheres. It would be interesting to know if the value of $c_{i}^{k}$ is the smallest possible power of $p$ for which there is such a factorization. Since $c_{i}^{k}$ depends so strongly on the action of the Steenrod algebra on the generators of $H^{*}\left(B_{i}^{k}\right)$, it is likely that it is best possible.

Example 5.3. As the proof of Theorem 5.1 is lengthy, it may be helpful to focus on the rank 2 case. Observe that $A_{i}^{2}$ has two cells, in degrees $2 i+1$ and $2 i+q+1$. There are two cases, depending on $i$. If $c_{i}^{2}=1$, then the two cells of $A_{i}^{2}$ are connected in cohomology by the Steenrod operation $\mathcal{P}^{1}$. The statement of Theorem 5.1 then claims there is a homotopy commutative diagram

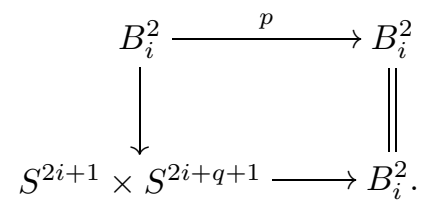




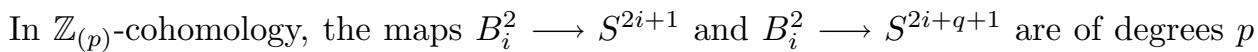
and 1 respectively, and the maps $S^{2 i+1} \longrightarrow B_{i}^{2}$ and $S^{2 i+q+1} \longrightarrow B_{i}^{2}$ are of degrees 1 and $p$ respectively. If $c_{i}^{2}=0$, then the two cells of $A_{i}^{2}$ are not connected in cohomology by the Steenrod operation $\mathcal{P}^{1}$, and so for low dimensional reasons, $A_{i}^{2} \simeq S^{2 i+1} \vee S^{2 i+q+1}$. The statement of Theorem 5.1 then claims there is a homotopy commutative diagram

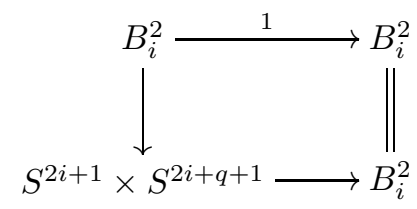

where all the maps to and from spheres are degree 1 in cohomology. In particular, this implies $B_{i}^{2} \simeq S^{2 i+1} \times S^{2 i+q+1}$.

Before proceeding with the proof of Theorem 5.1 we record a particular instance of Lemma 3.6 which will be used repeatedly.

Lemma 5.4. Let $A_{i}^{k-1} \longrightarrow A_{i}^{k}$ be the skeletal inclusion. Then there is a homotopy commutative diagram

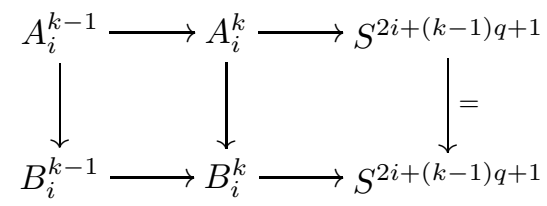

where the top row is a homotopy cofibration, the bottom row is a homotopy fibration of $H$-spaces and $H$-maps, and the vertical maps are the inclusions into the universal spaces.

Proof. Apply Lemma 3.6 to the homotopy cofibration $A_{i}^{k-1} \rightarrow A_{i}^{k} \rightarrow S^{2 i+(k-1) q+1}$, noting that by Example 3.3 the universal space for $S^{2 i+(k-1) q+1}$ is itself.

Iterating Lemma 5.4 applied to the homotopy cofibrations $A_{i}^{t-1} \longrightarrow A_{i}^{t} \longrightarrow$ $S^{2 i+(t-1) q+1}$ for $1 \leq t \leq k$ gives:

Corollary 5.5. The space $B_{i}^{k}$ is spherically resolved by multiplicative homotopy fibrations $B_{i}^{t-1} \longrightarrow B_{i}^{t} \longrightarrow S^{2 i+(t-1) q+1}$ for $1 \leq t \leq k$.

Proof of Theorem [5.1. We induct on the rank of $B_{i}^{k}$. The $k=0$ case is a tautology as $B_{i}^{1}=S^{2 i+1}$ and all the maps in the asserted diagram are identities. Assume the $(k-1)^{s t}$ case holds. Two possibilities exist at each stage of the induction, depending on whether or not consecutive cells in $A_{i}^{k}$ are connected by the Steenrod operation $\mathcal{P}^{1}$ in cohomology. The setup for both cases is the same.

Setup: Consider the homotopy cofibration

$$
S^{2 i+(k-1) q} \longrightarrow A_{i}^{k-1} \longrightarrow A_{i}^{k}
$$

which attaches the top cell of $A_{i}^{k}$. Let $\psi$ be the composite

$$
\psi: A_{i}^{k-1} \longrightarrow B_{i}^{k-1} \longrightarrow \prod_{t=1}^{k-1} S^{2 i+(t-1) q+1}
$$


where the left map is the inclusion and the right map is from the inductively assumed factorization of the $p^{c_{i}^{k-1}}$-power map on $B_{i}^{k-1}$. Let $\theta$ be the composite

$$
\theta: S^{2 i+(k-1) q} \longrightarrow A_{i}^{k-1} \stackrel{\psi}{\longrightarrow} \prod_{t=1}^{k-1} S^{2 i+(t-1) q+1} .
$$

Note that the projection of $\theta$ to $S^{2 i+(t-1) q+1}$ represents an element in $\pi_{2 i+(k-1) q}\left(S^{2 i+(t-1) q+1}\right)$. Since $k<p-2$, Toda's [To] calculations of the low dimensional unstable homotopy groups of spheres show that $\pi_{2 i+(k-1) q}\left(S^{2 i+(t-1) q+1}\right) \cong$ $\mathbb{Z} / p \mathbb{Z}$, with a generator represented by the stable map $\alpha_{k-t}$. So $\theta=\prod_{t=1}^{k-1} s_{t} \cdot \alpha_{k-t}$ for constants $s_{t} \in \mathbb{Z} / p \mathbb{Z}$. In particular, focus on $t=k-1$. Then $\theta$ projects to $s_{k-1} \cdot \alpha_{1}$. The map $\alpha_{1}$ is detected in cohomology by the Steenrod operation $\mathcal{P}^{1}$. To be precise, we have $H^{*}\left(A_{i}^{k}\right) \cong \Lambda\left(x_{2 i+1}, \ldots, x_{2 i+(k-1) q+1}\right)$. If $\mathcal{P}^{1}\left(x_{2 i+(k-2) q+1}\right)=$ $u \cdot x_{2 i+(k-1) q+1}$ for some unit $u \in \mathbb{Z} / p \mathbb{Z}$, then $s_{k-1}$ is also a unit in $\mathbb{Z} / p \mathbb{Z}$. If $\mathcal{P}^{1}\left(x_{2 i+(k-2) q+1}\right)=0$, then $s_{k-1}=0$ as well.

Case 1: $\mathcal{P}^{1}\left(x_{2 i+(k-2) q+1}\right)=u \cdot x_{2 i+(k-1) q+1}$ for some unit $u \in \mathbb{Z} / p \mathbb{Z}$, and so $s_{k-1} \neq 0$. Then $\theta$ is nontrivial and has order $p$. Thus there is a homotopy pushout (1)

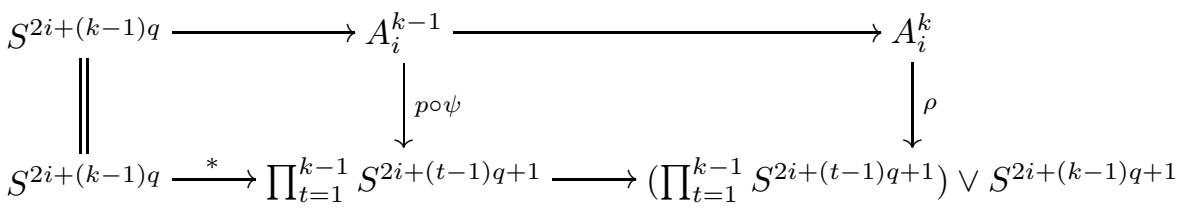

which defines the map $\rho$. Observe that $\rho$ followed by the pinch map to $S^{2 i+(k-1) q+1}$ is homotopic to the pinch map from $A_{i}^{k}$ onto its top dimensional cell, and so is degree 1 in cohomology. By the definition of $\psi$ and the inductive hypothesis, the composite

$$
A_{i}^{k-1} \stackrel{\psi}{\longrightarrow} \prod_{t=1}^{k-1} S^{2 i+(t-1) q+1} \rightarrow S^{2 i+(t-1) q+1}
$$

has degree $p^{c_{i}^{k-1}-c_{i}^{t}}$ in $\mathbb{Z}_{(p)}$-cohomology, so the composite

$$
A_{i}^{k} \stackrel{\rho}{\longrightarrow}\left(\prod_{t=1}^{k-1} S^{2 i+(t-1) q+1}\right) \vee S^{2 i+(k-1) q+1} \rightarrow S^{2 i+(t-1) q+1}
$$

has degree $p^{c_{i}^{k-1}+c_{i}^{t}+1}$. As we assumed that $\mathcal{P}^{1}\left(x_{2 i+(k-2) q+1}\right) \neq 0$ to begin this case, we have $c^{k-1}+1=c_{i}^{k}$ since $c_{i}^{k}$ counts the number of nonzero $\mathcal{P}^{1}$ operations connecting the generators of $\widetilde{H}^{*}\left(A_{i}^{k}\right)$.

Step 1: To use pushout (11), we claim that the composites

$$
A_{i}^{k-1} \longrightarrow A_{i}^{k} \longrightarrow B_{i}^{k} \stackrel{p^{c_{i}^{k}}}{\longrightarrow} B_{i}^{k}
$$

and

$$
A_{i}^{k-1} \stackrel{p \circ \psi}{\longrightarrow} \prod_{t=1}^{k-1} S^{2 i+(t-1) q+1} \longrightarrow B_{i}^{k-1} \longrightarrow B_{i}^{k}
$$

are homotopic. First, $\psi$ is the composite $A_{i}^{k-1} \longrightarrow B_{i}^{k-1} \longrightarrow \prod_{t=1}^{k-1} S^{2 i+(t-1) q+1}$, and by inductive hypothesis the composite $B_{i}^{k-1} \longrightarrow \prod_{t=1}^{k-1} S^{2 i+(t-1) q+1} \longrightarrow B_{i}^{k-1}$ 
is homotopic to $p^{c_{i}^{k-1}}$, so we see that (3) is homotopic to

$$
A_{i}^{k-1} \longrightarrow B_{i}^{k-1} \stackrel{p^{c_{i}^{k-1}+1}}{\longrightarrow} B_{i}^{k-1} \longrightarrow B_{i}^{k} .
$$

As mentioned, in this case $c_{i}^{k-1}+1=c_{i}^{k}$. Applying Lemma 5.4 to the inclusion $A_{i}^{k-1} \longrightarrow A_{i}^{k}$, we can assume the corresponding map $B_{i}^{k-1} \longrightarrow B_{i}^{k}$ of universal spaces is an $H$-map. Thus (4) is homotopic to the composite

$$
A_{i}^{k-1} \longrightarrow B_{i}^{k-1} \longrightarrow B_{i}^{k} \stackrel{p^{c_{i}^{k}}}{\longrightarrow} B_{i}^{k} .
$$

Lemma 5.4also says that $A_{i}^{k-1} \longrightarrow B_{i}^{k-1} \longrightarrow B_{i}^{k}$ is homotopic to $A_{i}^{k-1} \longrightarrow A_{i}^{k} \longrightarrow$ $B_{i}^{k}$, so (5) is homotopic to (2). Hence (2) is homotopic to (3). The homotopy pushout in (1) then gives a pushout map $b:\left(\prod_{t=1}^{k-1} S^{2 i+(t-1) q+1}\right) \vee S^{2 i+(k-1) q+1} \longrightarrow$ $B_{i}^{k}$ and a homotopy commutative diagram

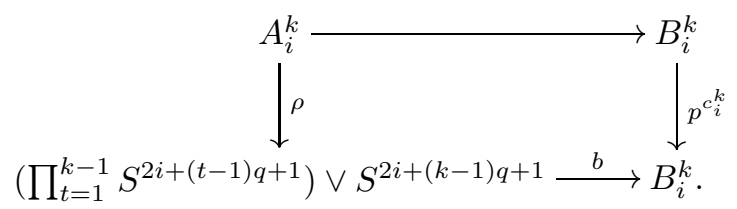

Step 2: Let $b_{1}, b_{2}$ be the restrictions of $b$ to $\prod_{t=1}^{k-1} S^{2 i+(t-1) q+1}$ and $S^{2 i+(k-1) q+1}$ respectively. The construction of $b$ in Step 1 implies that $b_{1}$ is an $H$-map, as it is the composite of the $H$-maps $\prod_{t=1}^{k-1} S^{2 i+(t-1) q+1} \longrightarrow B_{i}^{k-1} \longrightarrow B_{i}^{k}$. Example 3.3 shows that any map from an odd dimensional sphere to a homotopy associative, homotopy commutative $H$-space is homotopic to an $H$-map. Since $B_{i}^{k}$ is homotopy associative and homotopy commutative, $b_{2}$ is therefore homotopic to an $H$-map. Clearly $b$ factors through the composite

$$
b_{1} \cdot b_{2}:\left(\prod_{t=1}^{k-1} S^{2 i+(t-1) q+1}\right) \times S^{2 i+(k-1) q+1} \stackrel{b_{1} \times b_{2}}{\longrightarrow} B_{i}^{k} \times B_{i}^{k} \stackrel{\mu}{\longrightarrow} B_{i}^{k} .
$$

As $B_{i}^{k}$ is homotopy associative and homotopy commutative, $\mu$ is an $H$-map. Thus $b_{1} \cdot b_{2}$ is a composite of $H$-maps and so is an $H$-map. Hence there is a homotopy commutative diagram

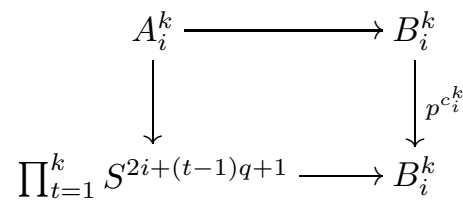

where the map along the lower row is an $H$-map.

Step 3: Let $\phi: A_{i}^{k} \longrightarrow \prod_{t=1}^{k} S^{2 i+(k-1) q+1} \longrightarrow B_{i}^{k}$ be the lower direction around the square at the end of Step 2. The homotopy commutativity of the square implies that $B_{i}^{k} \stackrel{p^{c_{i}^{k}}}{\longrightarrow} B_{i}^{k}$ is an $H$-map which extends $\phi$. On the other hand, $\prod_{t=1}^{k} S^{2 i+(k-1) q+1}$ is homotopy associative and homotopy commutative and $B_{i}^{k}$ is universal for $A_{i}^{k}$, so the map $A_{i}^{k} \longrightarrow \prod_{t=1}^{k} S^{2 i+(k-1) q+1}$ extends to an $H$ map $B_{i}^{k} \longrightarrow \prod_{t=1}^{k} S^{2 i+(k-1) q+1}$. Thus the composite of $H$-maps $\varphi: B_{i}^{k} \longrightarrow$ $\prod_{t=1}^{k} S^{2 i+(k-1) q+1} \longrightarrow B_{i}^{k}$ also extends $\phi$. The universal property says there is 
a unique $H$-map $B_{i}^{k} \longrightarrow B_{i}^{k}$ extending $\phi$, and so $\varphi \simeq p^{c_{i}^{k}}$, giving a homotopy commutative diagram of $H$-maps

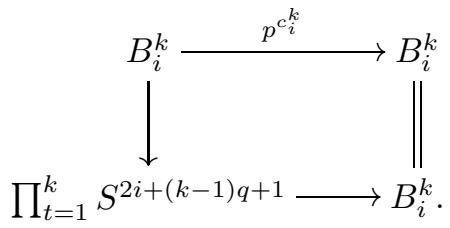

All that remains to complete the inductive step is to check the degree assertions. It suffices to check that these hold when the lower direction around the previous square is restricted to $A_{i}^{k}$. By construction, this restriction is homotopic to the composite $A_{i}^{k} \stackrel{\rho}{\longrightarrow}\left(\prod_{t=1}^{k-1} S^{2 i+(t-1) q+1}\right) \vee S^{2 i+(k-1) q+1} \stackrel{b}{\longrightarrow} B_{i}^{k}$. The degree assertions now follow from the degree properties of $\rho$ stated immediately after (11).

Case 2: $\mathcal{P}^{1}\left(x_{2 i+(k-2) q+1}\right)=0$, and so $s_{k-1}=0$. If $A_{i}^{k}$ has two cells, then this case has been dealt with in Example 5.3. So we may assume that $A_{i}^{k}$ has at least three cells, meaning $k>2$. As $s_{k-1}=0$, the projection of $\theta$ to $S^{2 i+(k-2) q+1}$ is null homotopic.

Step 1: Since $B_{i}^{k}$ is spherically resolved there is a homotopy fibration $B_{i}^{k-1} \longrightarrow$ $B_{i}^{k} \longrightarrow S^{2 i+(k-1) q+1}$. The triviality of $\theta$ when projected to $S^{2 i+(k-2) q+1}$ then implies there is a lift

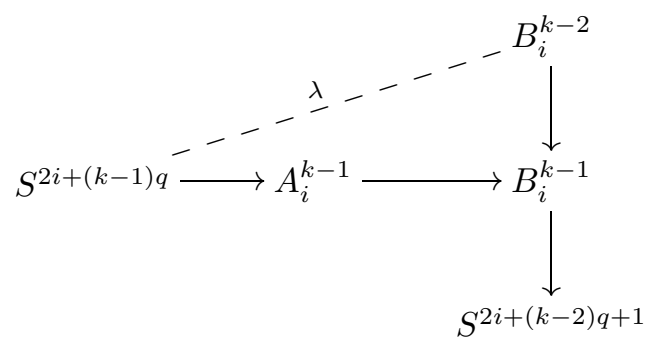

for some map $\lambda$.

Step 2: We claim that the diagram

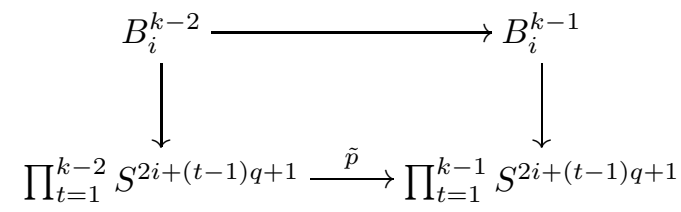

homotopy commutes. Here, the two vertical maps are the inductively assumed $H$ maps and $\tilde{p}$ is multiplication by $p$ on $\prod_{t=1}^{k-2} S^{2 i+(t-1) q+1}$ followed by the inclusion into $\prod_{t=1}^{k-1} S^{2 i+(t-1) q+1}$.

To prove the claim, first recall from when $c_{i}^{k}$ was defined that there is at most one instance of two consecutive cells in $A_{i}^{k}$ not being connected by a $\mathcal{P}^{1}$ in cohomology. The hypothesis $\mathcal{P}^{1}\left(x_{2 i+(k-2) q+1}\right)=0$ to start this case then implies that $\mathcal{P}^{1}\left(x_{2 i+(k-3) q+1}\right) \neq 0$, and so the preceding step in the induction going from rank $k-2$ to rank $k-1$ was subject to Case 1 . We assume this from here on. Observe that $\prod_{t=1}^{k-1} S^{2 i+(t-1) q+1}$ is homotopy associative and homotopy commutative, and that all maps in (6) are $H$-maps. Since $B_{i}^{k-2}$ is universal for $A_{i}^{k-2}$, it suffices to show 
that (6) homotopy commutes when restricted to $A_{i}^{k-2}$. Consider the composites

$$
A_{i}^{k-2} \longrightarrow B_{i}^{k-2} \longrightarrow \prod_{t=1}^{k-2} S^{2 i+(t-1) q+1} \stackrel{\tilde{p}}{\longrightarrow} \prod_{t=1}^{k-1} S^{2 i+(t-1) q+1}
$$

and

$$
A_{i}^{k-2} \longrightarrow B_{i}^{k-2} \longrightarrow B_{i}^{k-1} \longrightarrow \prod_{t=1}^{k-1} S^{2 i+(t-1) q+1} .
$$

We need to show that (77) is homotopic to (8). First, the combination of the left and middle maps in (7) is the definition of $\psi$ (in the $k-2$ case), so (7) is homotopic to the composite

$$
A_{i}^{k-2} \stackrel{p \circ \psi}{\longrightarrow} \prod_{t=1}^{k-2} S^{2 i+(t-1) q+1} \longrightarrow \prod_{t=1}^{k-1} S^{2 i+(t-1) q+1} .
$$

Clearly, this is homotopic to the composite

$$
\begin{aligned}
\phi_{1}: A_{i}^{k-2} \stackrel{p \circ \psi}{\longrightarrow} \prod_{t=1}^{k-2} S^{2 i+(t-1) q+1} \longrightarrow\left(\prod_{t=1}^{k-2} S^{2 i+(t-1) q+1}\right) \vee S^{2 i+(k-1) q+1} \\
\hookrightarrow \prod_{t=1}^{k-1} S^{2 i+(t-1) q+1} .
\end{aligned}
$$

On the other hand, since $B_{i}^{k-2}$ and $B_{i}^{k-1}$ are universal for $A_{i}^{k-2}$ and $A_{i}^{k-1}$ respectively, applying Lemma 5.4 to the inclusion $A_{i}^{k-2} \longrightarrow A_{i}^{k-1}$ implies that (8) is homotopic to the composite

$$
A_{i}^{k-2} \longrightarrow A_{i}^{k-1} \longrightarrow B_{i}^{k-1} \longrightarrow \prod_{t=1}^{k-1} S^{2 i+(t-1) q+1}
$$

The map $B_{i}^{k-1} \longrightarrow \prod_{t=1}^{k-1} S^{2 i+(t-1) q+1}$ was constructed in Case 1 as an extension of the composite $A_{i}^{k-1} \stackrel{\rho}{\longrightarrow}\left(\prod_{t=1}^{k-2} S^{2 i+(t-1) q+1}\right) \vee S^{2 i+(k-1) q+1} \longrightarrow \prod_{t=1}^{k-1} S^{2 i+(t-1) q+1}$. Thus (8) is homotopic to the composite

$$
\phi_{2}: A_{i}^{k-2} \longrightarrow A_{i}^{k-1} \stackrel{\rho}{\longrightarrow}\left(\prod_{t=1}^{k-2} S^{2 i+(t-1) q+1}\right) \vee S^{2 i+(k-1) q+1} \hookrightarrow \prod_{t=1}^{k-1} S^{2 i+(t-1) q+1} .
$$

Thus to show (7) is homotopic to (8) it suffices to show that $\phi_{1} \simeq \phi_{2}$. But this is exactly given by the homotopy pushout

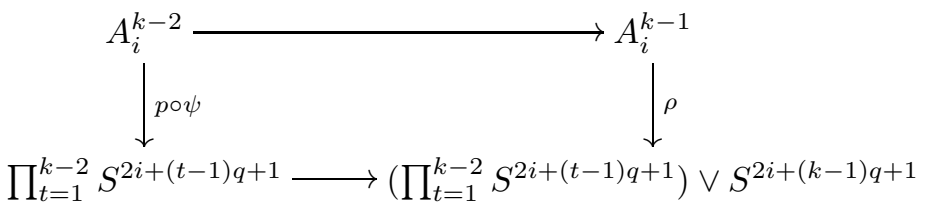

which began the proof of Case 1 (in the step from rank $k-2$ to rank $k-1$ ). Thus (6) homotopy commutes.

Step 3: We return to the lift $\lambda$ in Step 1. Again using Toda's calculations of the low dimensional homotopy groups of spheres, the composite $S^{2 i+(k-1) q} \stackrel{\lambda}{\longrightarrow}$ $B_{i}^{k-2} \longrightarrow \prod_{t=1}^{k-2} S^{2 i+(t-1) q+1}$ is homotopic to $\prod_{t=1}^{k-2} r_{t} \cdot \alpha_{k-t}$ for constants $r_{t} \in \mathbb{Z} / p \mathbb{Z}$. 
But then $\tilde{p} \circ\left(\prod_{t=1}^{k-2} r_{t} \cdot \alpha_{k-t}\right)$ is null homotopic. The homotopy commutativity of (6) therefore shows that the composite $S^{2 i+(k-1) q} \stackrel{\lambda}{\longrightarrow} B_{i}^{k-2} \longrightarrow B_{i}^{k-1} \longrightarrow$ $\prod_{t=1}^{k-1} S^{2 i+(t-1) q+1}$ is null homotopic. The definition of $\lambda$ then says that the composite $S^{2 i+(k-1) q} \longrightarrow A_{i}^{k-1} \longrightarrow B_{i}^{k-1} \longrightarrow \prod_{t=1}^{k-1} S^{2 i+(t-1) q+1}$ is null homotopic. So there is a homotopy pushout

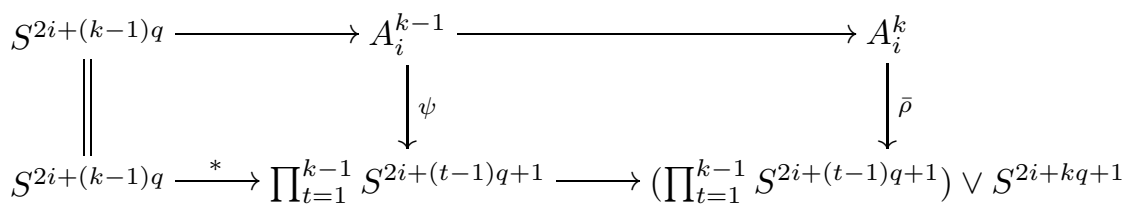

which defines the map $\bar{\rho}$. Observe that this pushout is similar to the one which began the proof in Case 1, except that the map $A_{i}^{k-1} \longrightarrow \prod_{t=1}^{k-1} S^{2 i+(t-1) q+1}$ here is $\psi$, while in Case 1 it is $p \circ \psi$. Proceeding now as in Case 1 , we replace (3) by

$$
A_{i}^{k-1} \stackrel{\psi}{\longrightarrow} \prod_{t=1}^{k-1} S^{2 i+(t-1) q+1} \longrightarrow B_{i}^{k-1} \longrightarrow B_{i}^{k}
$$

Arguing as before, with $\psi$ in (9) instead of $p \circ \psi$ in (3), we see that (9) is homotopic to the composite

$$
A_{i}^{k-1} \longrightarrow B_{i}^{k-1} \stackrel{p_{i}^{c_{i}^{k-1}}}{\longrightarrow} B_{i}^{k-1} \longrightarrow B_{i}^{k}
$$

which in turn is homotopic to the composite

$$
A_{i}^{k-1} \longrightarrow B_{i}^{k-1} \longrightarrow B_{i}^{k \stackrel{c_{i}^{k-1}}{\longrightarrow}} B_{i}^{k}
$$

As we assumed that $\mathcal{P}^{1}\left(x_{2 i+(k-2) q+1}\right)=0$ to begin this case, we have $c_{i}^{k}=c_{i}^{k-1}$ since, by definition, $c_{i}^{k}$ counts the number of nonzero $\mathcal{P}^{1}$ operations in $\widetilde{H}^{*}\left(A_{i}^{k}\right)$. Hence (10) equals (2), and so (9) is homotopic to (2). The remainder of the proof now follows as in Case 1.

Remark 5.6. The maps $S^{2 i+(k-1) q+1} \longrightarrow B_{i}^{k}$ of degree $p^{c_{i}^{k}}$ in Theorem [5.1] are "characteristic" maps. Recall that there is a canonical characteristic map $S^{2 n-1} \longrightarrow$ $S U(n)$ which, when composed with the quotient map $S U(n) \longrightarrow S^{2 n-1}$, has degree $(n-1) !$. As we are localized at $p$, we only care about the number of times $p$ divides $(n-1)$ !, which we write as $\nu_{p}((n-1) !)$. The decomposition $S U(n) \simeq \prod_{i=1}^{p-1} B_{i}$ results in a map $B_{i}^{k} \longrightarrow S^{2 i+(k-1) q+1}$ and a "characteristic" map $S^{2 i+(k-1) q+1} \longrightarrow B_{i}^{k}$ such that the composite $S^{2 i+(k-1) q+1} \longrightarrow B_{i}^{k} \longrightarrow S^{2 i+(k-1) q+1}$ is homotopic to $p^{\nu_{p}((i+(k-1)(p-1)) !)}$. With $1 \leq i \leq p-1$ and $0 \leq k \leq p-3$, it is easy to see that $c_{i}^{k}=\nu_{p}((i+(k-1)(p-1)) !)$. So the $H$-map $S^{2 i+(k-1) q+1} \longrightarrow B_{i}^{k}$ of Theorem 5.1 can be regarded as a particularly well-behaved choice of a characteristic map.

Proof of Theorem 1.3. We are considering the factors $B_{i}(G)$ of the decomposition $G \simeq \prod_{i=1}^{p-1} B_{i}(G)$ in Theorem 1.1. Each $B_{i}(G)$ is universal for a space $A_{i}(G)$, which is explicitly described in the proof of Theorem 2.1. In particular, when $G=S U(n)$, then $A(G)=\Sigma \mathbb{C} P^{n-1}$ and so $A_{i}(G)$ is a wedge summand of $\Sigma \mathbb{C} P^{n-1}$. Thus $A_{i}(S U(n))=A_{i}^{k} \in \mathcal{C}$ with $1 \leq i \leq p-1$ and $1 \leq k \leq p-3$. The factorization in Theorem 1.3 is therefore a special case of Theorem [5.1. Here, $r_{i}(S U(n))=c_{i}^{k}$, and this is explicitly described in Remark 5.6. 
As described in the proof of Theorem 2.1. the nonsphere factors of $G$ for the other Lie groups are factors of $S U(n)$ for $1 \leq n \leq(p-1)(p-3)$, except for some of the rank two factors of the exceptional groups, and the factor homotopy equivalent to $\bar{B}(23,35,47,59)$ in $E_{7}$ at the prime 7 . Those $B_{i}(G)$ 's which are also factors of $S U(n)$ have already been taken care of. The factorization in Theorem 1.3 for the rank two factors of the exceptional groups is explicitly given in Example 5.3. The proof of Theorem 2.1 shows that $\bar{B}(23,35,47,59)$ at 7 is homologically generated by a retract of a stunted complex projective space $A$ with four cells, and $\mathcal{P}^{1}$ acts nontrivially on the 23, 35, 47 generators in cohomology. Theorem 5.1 then applies to the corresponding universal space $B$ and $c_{i}^{4}=3$.

\section{REFERENCES}

[B] R. Bott, A note on the Samelson product in the classical Lie groups, Comment. Math. Helv. 34 (1960), 245-256. MR0123330 (23:A658)

[C] F.R. Cohen, A short course in some aspects of classical homotopy theory, Lecture Notes in Math. 1286, Springer-Verlag (1987), 1-92. MR.922923 (89e:55027)

[CMN] F.R. Cohen, J.C. Moore, and J.A. Neisendorfer, The double suspension and exponents of the homotopy groups of spheres, Ann. of Math. 110 (1979), 549-565. MR/554384 (81c:55021)

[CN] F.R. Cohen and J.A. Neisendorfer, A construction of $p$-local $H$-spaces, pp. 351-359. Lecture Notes in Math. Vol. 1051, Springer, Berlin, 1984. MR764588 (86e:55011)

[CHZ] G. Cooke, J. Harper, and A. Zabrodsky, Torsion free $\bmod p H$-spaces of low rank, Topology 18 (1979), 349-359. MR551016 (80k:55032)

[DT] D.M. Davis and S.D. Theriault, Odd-primary homotopy exponents of simple compact Lie groups, submitted.

[G] B. Gray, On Toda's fibrations, Math. Proc. Camb. Phil. Soc. 97 (1985), 289-298. MR771822 (86i:55016)

[H] B. Harris, On the homotopy groups of the classical groups, Ann. of Math. 74 (1961), 407-413. MR0131278 (24:A1130)

[IK] K. Iriye and A. Kono, Mod $p$ retracts of $G$-product spaces, Math. Z. 190 (1985), 357-363. MR 806893 (88a:55017)

[J] I.M. James, Reduced Product Spaces, Ann. of Math. 62 (1955), 170-197. MR0073181 $(17: 396 \mathrm{~b})$

[JT] I.M. James and E. Thomas, Homotopy-abelian topological groups, Topology 1 (1962), 237-240. MR0149483 (26:6970)

[Mc] C.A. McGibbon, Homotopy commutativity in localized groups, Amer. J. Math 106 (1984), 665-687. MR745146 (86a:55011)

[MNT1] M. Mimura, G. Nishida, and H. Toda, Localization of $C W$-complexes and its applications, J. Math. Soc. Japan 23 (1971), 593-624. MR0295347(45:4413)

[MNT2] M. Mimura, G. Nishida, and H. Toda, Mod- $p$ decomposition of compact Lie groups, Publ. RIMS, Kyoto Univ 13 (1977), 627-680. MR0478187 (57:17675)

[MT] M. Mimura and H. Toda, Cohomology operations and the homotopy of compact Lie groups I, Topology 9 (1970), 317-336. MR0266237 (42:1144)

[NS] J.A. Neisendorfer and P.S. Selick, Some examples of spaces with or without exponents, Current trends in algebraic topology, Part 1, CMS Conf. Proc. 2, Amer. Math. Soc., 1982, 343-357. MR686124 (84b:55017)

[S1] P.S. Selick, Odd primary torsion in $\pi_{k}\left(S^{3}\right)$, Topology 17 (1978), 407-412. MR.516219 (80c:55010)

[S2] P.S. Selick, Space exponents for loop spaces of spheres, Stable and unstable homotopy theory, Fields Inst. Commun.

[Sp] W. Specht, Die linearen Beziehumgen zwischen höheren Kommutatoren, Math. Z. 51 (1948), 367-376. 19, Amer. Math. Soc., 1998, 279-283. MR0028301(10:425d)

[T] S.D. Theriault, The $H$-structure of low rank torsion free $H$-spaces, Quart. J. Math. Oxford 56 (2005), 403-415. MR2161254 
[To] H. Toda, On Iterated Suspensions I, J. Math. Kyoto Univ. 5 (1965), 87-142. MR0210130 (35:1024)

[W] F. Wever, Operatoren in Lieschen Ringen, J. Reine Angew. Math. 187 (1949), 44-55. MR0034397(11:579i)

Department of Mathematical Sciences, University of Aberdeen, Aberdeen AB24 3UE, United KingDOM

E-mail address: s.theriault@maths.abdn.ac.uk 